\title{
Fixed-point free circle actions on 4-manifolds
}

\author{
WEIMIN CHEN
}

\begin{abstract}
This paper is concerned with fixed-point free $\mathbb{S}^{1}$-actions (smooth or locally linear) on orientable 4-manifolds. We show that the fundamental group plays a predominant role in the equivariant classification of such 4-manifolds. In particular, it is shown that for any finitely presented group with infinite center there are at most finitely many distinct smooth (resp. topological) 4-manifolds which support a fixed-point free smooth (resp. locally linear) $\mathbb{S}^{1}$-action and realize the given group as the fundamental group. A similar statement holds for the number of equivalence classes of fixedpoint free $\mathbb{S}^{1}$-actions under some further conditions on the fundamental group. The connection between the classification of the $\mathbb{S}^{1}$-manifolds and the fundamental group is given by a certain decomposition, called a fiber-sum decomposition, of the $\mathbb{S}^{1}$ manifolds. More concretely, each fiber-sum decomposition naturally gives rise to a Z-splitting of the fundamental group. There are two technical results in this paper which play a central role in our considerations. One states that the Z-splitting is a canonical JSJ decomposition of the fundamental group in the sense of Rips and Sela. Another asserts that if the fundamental group has infinite center, then the homotopy class of principal orbits of any fixed-point free $\mathbb{S}^{1}$-action on the 4 -manifold must be infinite, unless the 4-manifold is the mapping torus of a periodic diffeomorphism of some elliptic 3-manifold.
\end{abstract}

57S15; 57M07, 57M50

\section{Introduction}

Locally linear $\mathbb{S}^{1}$-actions on oriented 4 -manifolds were classified by Fintushel up to orientation-preserving equivariant homeomorphisms (for smooth $\mathbb{S}^{1}$-actions the classification is up to orientation-preserving equivariant diffeomorphisms); see $[16 ; 17$; 18]. One associates to each locally linear $\mathbb{S}^{1}$-action a legally weighted 3 -manifold, which is the orbit space decorated with certain orbit-type data and a characteristic class of the $\mathbb{S}^{1}$-action. The equivariant classification of the $\mathbb{S}^{1}$-four-manifolds is then given by the isomorphism classes of the corresponding legally weighted 3-manifolds.

An important technique for studying locally linear $\mathbb{S}^{1}$-actions on 4 -manifolds is a replacement trick due to Pao [35]. Pao's trick allows one to trade a certain weighted 
circle in a legally weighted 3-manifold for a pair of fixed points, or to have the weighted circle deleted and a 3-ball removed from the legally weighted 3-manifold. (In particular, Pao's replacement trick applies only to locally linear $\mathbb{S}^{1}$-actions with a nonempty fixed-point set.) This procedure has the effect of replacing the given $\mathbb{S}^{1}$-action by another (nonequivalent) $\mathbb{S}^{1}$-action on the same 4 -manifold. Besides the construction of locally linear, nonlinear $\mathbb{S}^{1}$-actions on $\mathbb{S}^{4}$ in the original paper [35], the following are some of the further implications of Pao's trick when combined with the classification results of Fintushel in $[16 ; 17 ; 18]$ :

- If a 4-manifold $X$ admits a locally linear (resp. smooth) $\mathbb{S}^{1}$-action with a pair of fixed points or a fixed 2-sphere, then $X$ admits infinitely many nonequivalent locally linear (resp. smooth) $\mathbb{S}^{1}$-actions; see [35]. (There are many examples of such 4-manifolds, including a large class of simply connected 4-manifolds.)

- Modulo the 3-dimensional Poincaré conjecture (which is now resolved [36]), a simply connected, smooth $\mathbb{S}^{1}$-four-manifold is diffeomorphic to a connected sum of $\mathbb{S}^{4}, \pm \mathbb{C P}^{2}$, or $\mathbb{S}^{2} \times \mathbb{S}^{2}$; see [18], compare also [48].

- If an oriented 4-manifold with $b_{2}^{+} \geq 1$ admits a locally linear (resp. smooth) $\mathbb{S}^{1}$-action having at least one fixed point, then it contains a topologically (resp. smoothly) embedded, essential 2-sphere of nonnegative self-intersection; see Baldridge [3, Theorem 2.1]. ${ }^{1}$ In particular, the Hurwitz map $\pi_{2} \rightarrow H_{2}$ has infinite image. Baldridge's theorem gives a useful obstruction for the existence of $\mathbb{S}^{1}$-actions with fixed points, particularly for the smooth case as such a smoothly embedded 2-sphere constrains the Seiberg-Witten invariants of the 4-manifold; see [19].

In this paper we study fixed-point free $\mathbb{S}^{1}$-actions on orientable 4 -manifolds, either smooth or locally linear depending on which category (ie smooth or topological) we work in. The arguments are valid for both categories; for simplicity, we shall work mainly in the smooth category. Our results indicate that the equivariant classification of fixed-point free $\mathbb{S}^{1}$-actions, where there is a lack of Pao's replacement trick, is sharply different from that of $\mathbb{S}^{1}$-actions with fixed points. In particular, we show that under reasonable assumptions the fundamental group plays a predominant role in the equivariant classification of 4 -manifolds with a fixed-point free $\mathbb{S}^{1}$-action. We showcase this phenomenon with the following two theorems.

\footnotetext{
${ }^{1}$ Baldridge [3] works in the smooth category, but the arguments are valid in the locally linear category as well.
} 
Theorem 1.1 Let $X$ be an orientable 4-manifold such that

(i) the center of $\pi_{1}(X)$ is infinite cyclic,

(ii) $\pi_{1}(X)$ is single-ended and is not isomorphic to the fundamental group of a Klein bottle, and

(iii) any canonical JSJ decomposition of $\pi_{1}(X)$ contains a vertex subgroup which is not isomorphic to an HNN extension of a finite cyclic group.

Then there exists a constant $C>0$, depending only on $\pi_{1}(X)$, such that the number of equivalence classes of fixed-point free $\mathbb{S}^{1}$-actions (smooth or locally linear) on $X$ is bounded by $C$.

Theorem 1.2 Let $G$ be a finitely presented group with infinite center. There exists a constant $C>0$, depending only on $G$, such that the number of diffeomorphism classes (resp. homeomorphism classes) of orientable 4-manifolds admitting a fixed-point free, smooth (resp. locally linear) $\mathbb{S}^{1}$-action, whose fundamental group is isomorphic to $G$, is bounded by $C$.

Our approach to equivariant classification (resp. classification) of fixed-point free $\mathbb{S}^{1}$ four-manifolds differs from the traditional approach of legally weighted 3-manifolds (see Fintushel $[16 ; 17 ; 18]$ ) where, in our method, geometric group theory plays a prominent role. The central notion in our approach is a certain decomposition of the $\mathbb{S}^{1}$-manifolds which is called a fiber-sum decomposition; see Definition 1.3. Such a decomposition gives rise to a $Z$-splitting of the fundamental group of the manifold, and the central result of this paper states that the $Z$-splitting is a canonical JSJ decomposition of the fundamental group in the sense of Rips and Sela [38, Theorem 1.5]. We also point out that the methods of this paper are essentially different from those in Hillman [25], where homotopy/homeomorphism classifications of $\mathbb{S}^{1}$-bundles over certain 3-manifolds are given. In particular, the diffeomorphism classification result in Theorem 1.2 is not accessible by the surgery-theoretic techniques employed in Hillman [25].

The orbit map of a fixed-point free $\mathbb{S}^{1}$-action on an orientable 4-manifold defines a Seifert-type $\mathbb{S}^{1}$-fibration of the 4 -manifold, giving the orbit space a structure of a closed, orientable 3-dimensional orbifold whose singular set consists of a disjoint union of embedded circles, called singular circles. (Equivalently, the 4-manifold is the total space of a principal $\mathbb{S}^{1}$-bundle over the 3 -orbifold.) With this understood, the building blocks of a fiber-sum decomposition are oriented fixed-point free $\mathbb{S}^{1}$-fourmanifolds whose corresponding orbit space is an irreducible 3-orbifold. We shall call such $\mathbb{S}^{1}$-four-manifolds irreducible. Note that the orientation of the 4-manifold 
determines an orientation of the base 3-orbifold, as the fibers of the Seifert-type $\mathbb{S}^{1}$-fibration are canonically oriented.

Definition 1.3 (Fiber-sum decomposition) Let $X$ be a smooth orientable 4-manifold. Suppose we are given a finite set of smooth oriented 4 -manifolds $X_{i}, i \in I$, with the following significance.

(i) For each $i \in I$, there is a fixed-point free $\mathbb{S}^{1}$-action on $X_{i}$ with orbit map $\pi_{i}: X_{i} \rightarrow Y_{i}$ where $Y_{i}$ is irreducible.

(ii) There is a finite set $J$ such that, for each $j \in J$, there exists a pair of distinct points $y_{j, 1}, y_{j, 2} \in \bigsqcup_{i \in I} Y_{i}$ which have the same multiplicity if singular.

(iii) Let $F_{j, 1}$ and $F_{j, 2}$ be the fibers of $\bigsqcup_{i} \pi_{i}: \bigsqcup_{i} X_{i} \rightarrow \bigsqcup_{i} Y_{i}$ over $y_{j, 1}$ and $y_{j, 2}$, respectively. For each $j \in J$, there is an orientation-reversing but fiberwise orientation-preserving, fiber-preserving diffeomorphism $\phi_{j}: \partial N d\left(F_{j, 1}\right) \rightarrow$ $\partial N d\left(F_{j, 2}\right)$.

(iv) For any $i \in I, j \in J$, if $Y_{i}$ contains exactly one of the points $y_{j, 1}, y_{j, 2}$, say $y_{j, 1} \in Y_{i}$, then the homotopy class of the fiber $F_{j, 1}$ generates a proper subgroup of $\pi_{1}\left(X_{i}\right)$.

With the above understood, we say that $X$ admits a fiber-sum decomposition if there exists a diffeomorphism between $X$ and the oriented 4-manifold

$$
\bigsqcup_{i \in I} X_{i} \backslash \bigsqcup_{j \in J}\left(N d\left(F_{j, 1}\right) \sqcup N d\left(F_{j, 2}\right)\right) / \sim \bigsqcup_{j \in J} \phi_{j},
$$

and, given such a diffeomorphism, we say that $X$ is fiber-sum-decomposed into $X_{i}$ along $N_{j}$, where each $N_{j} \cong \mathbb{S}^{1} \times \mathbb{S}^{2}$ is the image of $\partial N d\left(F_{j, 1}\right)$ (or equivalently, $\left.\partial N d\left(F_{j, 2}\right)\right)$ in $X$. Furthermore, the irreducible $\mathbb{S}^{1}$-four-manifolds $X_{i}$ are called the factors of the fiber-sum decomposition.

Remarks The isotopy classification of diffeomorphisms of $\mathbb{S}^{1} \times \mathbb{S}^{2}$ is given by $\pi_{0}(O(2) \times O(3) \times \Omega O(3))$; see Hatcher [23]. In particular, there are two distinct isotopy classes of homologically trivial diffeomorphisms because of the factor $\pi_{0}(\Omega O(3))=$ $\pi_{1} \mathrm{SO}(3)=\mathbb{Z}_{2}$. However, the isotopy class of the diffeomorphism $\phi_{j}: \partial N d\left(F_{j, 1}\right) \rightarrow$ $\partial N d\left(F_{j, 2}\right)$ is uniquely determined because of the requirement that it be fiber-preserving.

It turns out that the class of 4-manifolds which admit a fiber-sum decomposition are precisely the smooth, fixed-point free $\mathbb{S}^{1}$-four-manifolds whose fundamental group has infinite center. In order to understand this, we recall that a fixed-point free $\mathbb{S}^{1}$-action is called injective (and so is the corresponding $\mathbb{S}^{1}$-four-manifold) if the homotopy 
class of the principal orbits has infinite order. With this understood, note that in Definition 1.3 each 3 -orbifold $Y_{i}$ is irreducible. It follows easily that the $\mathbb{S}^{1}$-action on each $X_{i}$ must be injective. Moreover, it is clear that the $\mathbb{S}^{1}$-actions on $X_{i}$ descend to a fixed-point free $\mathbb{S}^{1}$-action on $X$, which is also injective. On the other hand, given any injective $\mathbb{S}^{1}$-action, the orbit space (as a 3 -orbifold) admits a certain kind of spherical decompositions which are called reduced (see Lemma 2.4 for details), and any such spherical decomposition naturally gives rise to a fiber-sum decomposition of the 4-manifold (for more details see the proof of Theorem 1.4).

In summary, a 4-manifold admits a fiber-sum decomposition if and only if it admits an injective fixed-point free $\mathbb{S}^{1}$-action. Note that the homotopy class of the principal orbits of the $\mathbb{S}^{1}$-action lies in the center of the fundamental group of the 4-manifold. In particular, $\pi_{1}$ of an injective fixed-point free $\mathbb{S}^{1}$-four-manifold has infinite center. The converse is given in the following theorem.

Theorem 1.4 Let $X$ be a smooth (resp. locally linear), fixed-point free $\mathbb{S}^{1}$-fourmanifold whose fundamental group has infinite center. Then the $\mathbb{S}^{1}$-action must be injective unless $X$ is diffeomorphic (resp. homeomorphic) to the mapping torus of a periodic diffeomorphism of some elliptic 3-manifold. As a consequence, any smooth, fixed-point free $\mathbb{S}^{1}$-four-manifold whose $\pi_{1}$ has infinite center admits a fibersum decomposition.

Note that in the case where $X$ is diffeomorphic to the mapping torus of a periodic diffeomorphism of some elliptic 3-manifold, $X$ admits another fixed-point free $\mathbb{S}^{1}$-action which is injective. So in any event, the 4-manifold admits a fiber-sum decomposition.

We remark that the fundamental group of a smooth, fixed-point free $\mathbb{S}^{1}$-four-manifold with nontrivial Seiberg-Witten invariant must have infinite center; see [11; 12].

With the preceding understood, the main theme of this paper is to recover the fiber-sum decompositions of an injective $\mathbb{S}^{1}$-four-manifold from its fundamental group. The main results are summarized in Theorems 1.5 and 1.6 below.

In order to describe the results, observe that given any fiber-sum decomposition of $X$ into factors $X_{i}$ along $N_{j}$, there is an associated finite graph of groups where the vertex groups and edge groups are given by $\pi_{1}\left(X_{i}\right)$ and $\pi_{1}\left(N_{j}\right)$, respectively, such that $\pi_{1}(X)$ is isomorphic to the fundamental group of the graph of groups. Such a presentation of $\pi_{1}(X)$ is called a $Z$-splitting as each edge group $\pi_{1}\left(N_{j}\right)$ is infinite cyclic. An in-depth study of Z-splittings of single-ended finitely generated groups was given in [38] by Rips and Sela; in particular, they showed the existence of certain "universal" Z-splittings for each single-ended finitely presented group, which are called canonical JSJ decompositions. 
Theorem 1.5, which is the main technical result of this paper, asserts that the Zsplitting associated to a fiber-sum-decomposition is a canonical JSJ decomposition of the fundamental group.

Theorem 1.5 Let $X$ and $X^{\prime}$ be smooth 4-manifolds which are fiber-sum-decomposed into $X_{i}$ along $N_{j}$ and $X_{i^{\prime}}^{\prime}$ along $N_{j^{\prime}}^{\prime}$, respectively. Suppose $\pi_{1}(X)$ and $\pi_{1}\left(X^{\prime}\right)$ are single-ended and are not isomorphic to the fundamental group of a 2-torus or a Klein bottle. Then the following hold.

(1) The Z-splitting of $\pi_{1}(X)$ associated to the given fiber-sum-decomposition of $X$ is a canonical JSJ decomposition. ${ }^{2}$

(2) Assume further that the submanifolds $N_{j}$ and $N_{j^{\prime}}^{\prime}$ are null-homologous in $X$ and $X^{\prime}$, respectively, and let $\alpha: \pi_{1}(X) \rightarrow \pi_{1}\left(X^{\prime}\right)$ be any isomorphism. Then after modifying the embeddings of $N_{j}$ and $N_{j^{\prime}}^{\prime}$ by fiber-preserving isotopies if necessary, $\alpha: \pi_{1}(X) \rightarrow \pi_{1}\left(X^{\prime}\right)$ may be enhanced to an isomorphism between the $Z$-splittings of $\pi_{1}(X)$ and $\pi_{1}\left(X^{\prime}\right)$ associated to the new fiber-sum decompositions of $X$ and $X^{\prime}$, respectively.

Remarks (1) Canonical JSJ decompositions are not unique as Z-splittings. Nevertheless, Theorem 1.5(1) implies that the number of factors $X_{i}$, the number of submanifolds $N_{j}$, as well as the conjugacy classes of subgroups $\pi_{1}\left(X_{i}\right)$ and $\pi_{1}\left(N_{j}\right)$, depend only on $\pi_{1}(X)$; see Proposition 3.5 for details. We shall also point out that in the course of the proof of Theorem 1.5, the group $\pi_{1}(X)$ is shown to have the property that it admits no hyperbolic-hyperbolic elementary Z-splittings; see Lemma 3.1.

(2) The stronger uniqueness in Theorem 1.5(2) corresponds to the uniqueness of canonical JSJ decompositions up to a sequence of slidings, conjugations, and conjugations of boundary monomorphisms. Such uniqueness has been established for torsion-free (Gromov) hyperbolic groups (see Sela [43, Theorem 1.7]), but remains open in general for single-ended finitely presented groups; see [38, page 106].

(3) The assumption that the submanifolds $N_{j}$ are null-homologous in $X$ is equivalent to the assumption that the underlying graph of the associated $\mathrm{Z}$-splitting of $\pi_{1}(X)$ is a tree. By Theorem 1.5(1), this assumption depends only on the group $\pi_{1}(X)$.

${ }^{2}$ There is an annoying collapse of terminology here as a canonical JSJ decomposition of $\pi_{1}(X)$ corresponds not to the JSJ decomposition of the base 3-orbifold, but to a reduced spherical decomposition of the 3-orbifold; see Lemma 2.4 . 
(4) It is worth pointing out that the considerations in this paper provide an almost ideal setting for the need for developing the algebraic theory of Rips and Sela on Z-splittings of single-ended finitely presented groups [38].

The next theorem, Theorem 1.6, is concerned with the building blocks of fiber-sum decompositions. In particular, it is shown that in most of the cases the diffeomorphism class of an irreducible $\mathbb{S}^{1}$-four-manifold is determined by the fundamental group. To state the result, we remark that a finitely generated group with infinite center is either single-ended or double-ended; see Lemma 4.1.

Theorem 1.6 Let $X$ and $X^{\prime}$ be irreducible $\mathbb{S}^{1}$-four-manifolds, and let $\alpha: \pi_{1}(X) \rightarrow$ $\pi_{1}\left(X^{\prime}\right)$ be any isomorphism.

(1) If $\pi_{1}(X)$ and $\pi_{1}\left(X^{\prime}\right)$ are single-ended, then there exists a diffeomorphism $\phi: X \rightarrow X^{\prime}$ such that $\phi_{*}=\alpha: \pi_{1}(X) \rightarrow \pi_{1}\left(X^{\prime}\right)$.

(2) If $\pi_{1}(X)$ and $\pi_{1}\left(X^{\prime}\right)$ are double-ended, then $X$ and $X^{\prime}$ are each the mappingtorus of a periodic diffeomorphism of an elliptic 3-manifold. Moreover, there exists a diffeomorphism $\phi: X \rightarrow X^{\prime}$ such that $\phi_{*}=\alpha: \pi_{1}(X) \rightarrow \pi_{1}\left(X^{\prime}\right)$, if the elliptic 3-manifold is not a lens space.

Finally, the cases which are not covered in Theorems 1.5 and 1.6, ie when $\pi_{1}(X)$ is isomorphic to the fundamental group of a 2-torus or a Klein bottle, are handled separately. In particular, we direct the reader's attention to two classification theorems of fixed-point free $\mathbb{S}^{1}$-four-manifolds. One is concerned with the situation where the center of $\pi_{1}$ is of rank greater than 1 ; the other is about the situation where $\pi_{1}$ is isomorphic to the $\pi_{1}$ of a Klein bottle. See Theorems 4.3 and 6.2 for more details.

With the preceding understood, Theorem 1.1 follows readily from Theorems 1.5 and 1.6. Theorem 1.2 also follows from Theorems 1.5 and 1.6 with the additional help of Theorems 1.4, 4.3 and 6.2.

Having reviewed the main theorems, we now give a few remarks about the technical aspect of this paper. Our arguments rely heavily on the recent advances in 3-dimensional topology, particularly those centered around the resolution of Thurston's Geometrization Conjecture (henceforth referred to as the Geometrization theorem; see [4; 36]; see also [14]). For instance, Lemma 5.2(which asserts that an orientable 3-orbifold is Seifert fibered if $\pi_{1}^{\text {orb }}$ has infinite center, and furthermore, if $\pi_{2}^{\text {orb }} \neq 0$, it is the mapping torus of a periodic diffeomorphism of a 2 -orbifold with finite $\pi_{1}^{\text {orb }}$ ) played a key role in the proofs of several theorems of this paper. The proof of this lemma involves several particular forms of the Geometrization theorem, which include the earlier work of 
Meeks and Scott [32] on finite group actions on Seifert 3-manifolds, the resolution of the Seifert fiber space conjecture due to Gabai [20] (and independently Casson and Jungreis [9]), as well as the more recent Orbifold theorem of Boileau, Leeb and Porti [4] and the resolution of Poincaré conjecture; see [36]. On the other hand, as we mentioned earlier, this paper also draws considerably from geometric group theory, particularly the work of Rips and Sela on Z-splittings of single-ended finitely presented groups; see [38].

Before ending the introduction, we point out a corollary of Theorem 1.4 which is of independent interest.

Corollary 1.7 Let $X$ be a 4-manifold whose fundamental group has infinite center. If $X$ admits a locally linear, fixed-point free $\mathbb{S}^{1}$-action, then there are no embedded $2-$ spheres with odd self-intersection in $X$. In particular, $X$ is minimal.

We end the introduction with the following questions, which are naturally suggested by the results of this paper (see $[44 ; 45 ; 46 ; 34 ; 47]$ for some relevant problems and results in dimension three).

Question 1.8 Let $X$ be an oriented, smooth, fixed-point free $\mathbb{S}^{1}$-four-manifold whose fundamental group has infinite center.

(1) Is the diffeomorphism type of $X$ determined by its homeomorphism type?

(2) Can one express the Seiberg-Witten invariant of $X$ in terms of topological invariants of the manifold?

The organization of the rest of the paper is as follows. In Section 2, we first review some basic definitions and facts about 2-orbifolds and 3-orbifolds, and then we prove several preliminary lemmas which will be used in later sections. Section 3 is devoted to the proof of Theorem 1.5; it begins with a brief review of the Bass-Serre theory of groups acting on trees (in particular, the definition of graph of groups and its fundamental group), as well as a review on the relevant part of the work of Rips and Sela in [38] concerning Z-splittings of single-ended finitely presented groups. The proof of Theorem 1.6 is given in Section 4, and so is the classification of fixed-point free $\mathbb{S}^{1}$-four-manifolds whose $\pi_{1}$ has a center of rank greater than 1 . Section 5 is devoted to Theorem 1.4; in particular, we prove the key lemma, Lemma 5.2, in this section. Corollary 1.7 asserting minimality of injective $\mathbb{S}^{1}$-four-manifolds is proven here as well. Section 6 contains the proofs of Theorems 1.1 and 1.2, as well as the classification of fixed-point free $\mathbb{S}^{1}$-four-manifolds whose $\pi_{1}$ is isomorphic to the $\pi_{1}$ of a Klein bottle.

Throughout this paper, we shall adopt the following notation: the center of a group $G$ is denoted by $z(G)$. 


\section{Recollections and preliminary lemmas}

For the reader's convenience, we shall begin by giving a brief review on the relevant definitions and basic facts about 2-orbifolds and 3-orbifolds; for more details, see eg $[41 ; 5]$. Recall first that an orbifold (not necessarily orientable) is called good if it is the quotient of a manifold by a properly discontinuous action of a discrete group; otherwise it is call bad. It is called very good if it is the quotient of a manifold by a finite group action. All orbifolds are assumed to be connected and closed (ie compact without boundary) unless mentioned otherwise.

An orientable 2-orbifold is given by a closed orientable surface as the underlying space, with isolated singular points where the local groups are cyclic, generated by a rotation. For a nonorientable 2-orbifold, if the underlying space has a nonempty boundary, the singular set will also contain the boundary of the underlying space, which is a polygon with local groups being either a reflection through a line in $\mathbb{R}^{2}$ or a dihedral group $D_{2 n}$ generated by two reflections through lines making an angle $\pi / n$. With this understood, a teardrop is a 2 -sphere with one singular point. A spindle is a 2-sphere with two singular points of different multiplicities (ie the orders of the local groups). A football is a 2-sphere with two singular points of the same multiplicity. A turnover is a 2-sphere with three singular points. Except for a teardrop or a spindle, all orientable $2-$ orbifolds are very good. An orientable 2-orbifold is called spherical (resp. toric, resp. hyperbolic) if it is the quotient of a 2-sphere (resp. 2-torus, resp. closed surface of genus $>1$ ) by a finite group. A 2-orbifold is spherical if and only if it is either a nonsingular sphere, a football, or a turnover with multiplicities $(2,2, n),(2,3,3)$, $(2,3,4)$, or $(2,3,5)$. The turnovers correspond to the quotient of 2 -sphere by the action of a dihedral group $D_{2 n}$ or one of the platonic groups $T_{12}, O_{24}$, or $I_{60}$.

All 2-suborbifolds in a 3-orbifold are assumed to be orientable. There is a special class of 3-orbifolds which are important for the considerations in this paper; these are the 3-orbifolds which do not contain any bad 2-suborbifolds. It is a consequence of the Geometrization theorem (see $[4 ; 31]$ ) that if a 3 -orbifold does not contain any bad 2 -suborbifolds, then it must be very good, ie it is the quotient of a 3-manifold by a finite group action. For simplicity, we shall call such a 3-orbifold good.

An orientable 3-orbifold (with or without boundary) is called spherical (resp. discal) if it is the quotient of the 3-sphere (resp. 3-ball) by a finite isometry group. A good 3 -orbifold is called irreducible if every spherical 2-suborbifold bounds a discal 3orbifold. An irreducible 3-orbifold is called atoroidal if it contains no essential toric 2-suborbifold. A 3-orbifold (not necessarily orientable) is called Seifert fibered if it is the total space of an orbifold bundle over a 2-orbifold (not necessarily orientable) with generic fiber a circle or a mirrored interval. (A mirrored interval is the quotient of a 
circle by an orientation-reversing involution.) It is easily seen that a generic fiber of an orientable Seifert fibered 3-orbifold must be a circle. Moreover, if the base 2-orbifold is orientable, then the singular set of the Seifert fibered 3-orbifold must consist of a union of fibers.

The rest of this section is occupied by a number of preliminary lemmas. The following lemma about the center of an amalgamated product or an HNN extension is well known to the experts. However, for the sake of completeness, we include a statement and a proof of the lemma here.

Lemma 2.1 (1) If $A \neq C \neq B$, then the center of $A *_{C} B$ is contained in $C$.

(2) Let $C \subset A$ be a subgroup and $\alpha: C \rightarrow A$ be an injective homomorphism, and let $A *_{C} \alpha$ denote the corresponding $H N N$ extension. Suppose $x \in z\left(A *_{C} \alpha\right)$. Then either $x \in C$, or $x$ is nontorsion, $C=A=\alpha(C)$, and $A *_{C} \alpha$ is isomorphic to $A *_{C} \alpha^{\prime}$ for some $\alpha^{\prime}: A \rightarrow A$ which is of finite order.

Proof For a proof of part (1), see Magnus, Karrass and Solitar [30], Corollary 4.5, page 211. We shall give a proof for part (2) here. An element of $A *_{C} \alpha$ can be uniquely represented by a reduced word; see eg Scott and Wall [42]. Lemma 2.1 is a direct consequence of this fact.

More concretely, recall that the group $A *_{C} \alpha$ is generated by elements of $A$ and a letter $t$ with additional relations $t c t^{-1}=\alpha(c)$ for all $c \in C$. We let $T$ and $T_{\alpha}$ be the sets of some fixed choices of representatives of the right cosets of $C$ and $\alpha(C)$ in $A$, respectively. Then a reduced word in $A *_{C} \alpha$ takes the form

$$
a_{1} t^{\epsilon_{1}} a_{2} t^{\epsilon_{2}} \cdots a_{n} t^{\epsilon_{n}} a_{n+1},
$$

where $\epsilon_{i}= \pm 1, a_{i} \in T$ if $\epsilon_{i}=+1, a_{i} \in T_{\alpha}$ if $\epsilon_{i}=-1$, and furthermore, $a_{i} \neq 1$ if $\epsilon_{i-1} \neq \epsilon_{i}$, and $a_{n+1}$ is allowed to be an arbitrary element of $A$.

Let $x=a_{1} t^{\epsilon_{1}} a_{2} t^{\epsilon_{2}} \cdots a_{n} t^{\epsilon_{n}} a_{n+1}$ be an element of the center (here $n=0$ represents the case where $x \in A$ ). If $n=0$, then by $t x=x t$ it is clear that $x=a_{n+1} \in C$ which obeys $\alpha(x)=x$. Suppose $n>0$. If $a_{1} \neq 1$, then the uniqueness of representation by reduced words implies that $t x \neq x t$, which is a contradiction. If $a_{1}=1$, then $t^{-\epsilon_{1}} x=x t^{-\epsilon_{1}}$ implies that $a_{2}=1$. Iterating this process, we see that $x=t^{l} a_{n+1}$ for some $0 \neq l \in \mathbb{Z}$. It follows from $t^{-1} x=x t^{-1}$ that $a_{n+1}=t a_{n+1} t^{-1}$, which implies that $a_{n+1} \in C$ and $\alpha\left(a_{n+1}\right)=a_{n+1}$. Furthermore, the commutativity of $t$ and $a_{n+1}$ also implies that $x=t^{l} a_{n+1}$ is nontorsion. To see $C=A=\alpha(C)$, note that if there is an $a \in T$ or $T_{\alpha}$ such that $a \neq 1$, then one has $a x \neq x a$ which is a contradiction. This implies that $C=A=\alpha(C)$. Now for any $c \in C$,

$$
t^{l} a_{n+1} \alpha^{l}(c)=x \alpha^{l}(c)=\alpha^{l}(c) x=\alpha^{l}(c) t^{l} a_{n+1}=t^{l} c a_{n+1},
$$


which implies that $a_{n+1} \alpha^{l}(c)=c a_{n+1}$ for any $c \in C=A$. Let $\alpha^{\prime}: A \rightarrow A$ be defined by $\alpha^{\prime}(c)=a_{n+1} \alpha(c) a_{n+1}^{-1}$. Then it follows from $\alpha\left(a_{n+1}\right)=a_{n+1}$ that

$$
\left(\alpha^{\prime}\right)^{l}(c)=a_{n+1} \alpha^{l}(c) a_{n+1}^{-1}=c, \quad \forall c \in A .
$$

Now note that $A *_{C} \alpha$ is isomorphic to $A *_{C} \alpha^{\prime}$ where $\alpha^{\prime}$ has finite order $l$. This completes the proof of the lemma.

For our purposes in this paper, it is important to understand the center of the fundamental group of a 2-orbifold or a 3-orbifold.

Lemma 2.2 Let $\Sigma$ be a 2-orbifold (not necessarily orientable) such that $z\left(\pi_{1}^{\text {orb }}(\Sigma)\right.$ ) is nontrivial. Then the following statements hold true.

(a) If $\Sigma$ is orientable, then it is either a football, a spindle with non-coprime multiplicities, a turnover with multiplicities $(2,2,2)$, or a nonsingular torus.

(b) If $\Sigma$ is nonorientable, then its orientable double cover $\widetilde{\Sigma}$ must lie in the following list: a nonsingular sphere, a teardrop, a spindle, a football, a turnover with multiplicities $(2,2,2)$, or a nonsingular torus. Moreover, $z\left(\pi_{1}^{\mathrm{orb}}(\Sigma)\right)$ is torsionfree if and only if $\widetilde{\Sigma}$ is a nonsingular torus.

Proof Suppose $\Sigma$ is orientable. If $\Sigma$ is bad, then it must be a spindle with non-coprime multiplicities because this is the only case where $\pi_{1}^{\text {orb }}(\Sigma)$ is nontrivial. Assume $\Sigma$ is good. If $\Sigma$ is spherical, then it must be a football or a turnover with multiplicities $(2,2,2)$, because the other groups, ie $D_{2 n}$ with $n \neq 2, T_{12}, O_{24}, I_{60}$, all have trivial center. If $\Sigma$ is toric, then it must be a nonsingular torus because the fundamental group of a toric turnover is centerless. Finally, $\Sigma$ can not be hyperbolic because a cocompact Fuchsian group has trivial center.

Suppose $\Sigma$ is nonorientable, and let $\widetilde{\Sigma}$ be the orientable 2 -orbifold which doubly covers $\Sigma$. Note that $\mathbb{Z}_{2}$ acts on $\widetilde{\Sigma}$ via deck transformations. We shall discuss the proof in two cases: (i) the deck transformations are free, (ii) the deck transformations are not free.

In case (i), the underlying space $|\Sigma|$ is a nonorientable, closed surface. We can decompose $|\Sigma|$ as the union of $\mathbb{R P}^{2} \backslash D^{2}$ and an orientable surface with one boundary component along their boundaries. Correspondingly, we have a decomposition of $\Sigma$ as the union of (nonsingular) $\mathbb{R P}^{2} \backslash D^{2}$ and an orientable 2-orbifold $\Sigma^{\prime}$ with one boundary component. It follows that $z\left(\pi_{1}^{\text {orb }}(\Sigma)\right)$ being nontrivial forces $\pi_{1}^{\text {orb }}\left(\Sigma^{\prime}\right)$ to be finite (see Lemma 2.1(1) and Lemma 2.2(a)), so that $\Sigma^{\prime}$ must be either a (nonsingular) $D^{2}$ or $D^{2} / \mathbb{Z}_{m}$ with $m>1$. This shows that the double cover $\widetilde{\Sigma}$ is either a (nonsingular) sphere or a football. 
In case (ii), if $z\left(\pi_{1}^{\text {orb }}(\widetilde{\Sigma})\right)$ is nontrivial, then we are done by part (a). Moreover, if $\widetilde{\Sigma}$ is a nonsingular torus, the fixed-point set of the deck transformation consists of a union of circles. Since the deck transformation is orientation-reversing, the Lefschetz fixed-point theorem implies that the action on $H_{1}(\tilde{\Sigma} ; \mathbb{R})$ must have eigenvalues +1 and -1 . It follows then that $z\left(\pi_{1}^{\text {orb }}(\Sigma)\right)=\mathbb{Z}$ in this case. If $z\left(\pi_{1}^{\text {orb }}(\widetilde{\Sigma})\right)$ is trivial, then $z\left(\pi_{1}^{\text {orb }}(\Sigma)\right)=\mathbb{Z}_{2}$ and acts on $\tilde{\Sigma}$ via deck transformations. Let $p$ be a fixed-point of the deck transformation. Since $\pi_{1}^{\text {orb }}(\widetilde{\Sigma}) \rightarrow \pi_{1}(|\widetilde{\Sigma}|)$ is surjective, the induced action of $z\left(\pi_{1}^{\text {orb }}(\Sigma)\right)=\mathbb{Z}_{2}$ on $\pi_{1}(|\tilde{\Sigma}|, p)$ must be trivial. This implies that the Lefschetz number of the action of $z\left(\pi_{1}^{\text {orb }}(\Sigma)\right)=\mathbb{Z}_{2}$ on $|\widetilde{\Sigma}|$ equals -2 times the genus of $|\widetilde{\Sigma}|$. The Lefschetz fixed-point theorem then implies that $|\widetilde{\Sigma}|$ has genus zero. If $\widetilde{\Sigma}$ is bad, then clearly we are done. If $\widetilde{\Sigma}$ is good, then it is the quotient of an orientable closed surface $\Sigma^{\prime}$ by a finite group. Note that $z\left(\pi_{1}^{\text {orb }}(\Sigma)\right)=\mathbb{Z}_{2}$ also acts on $\Sigma^{\prime}$ via deck transformations which are orientation-reversing. The same argument as above shows that $\Sigma^{\prime}$ must have genus zero. In other words, $\widetilde{\Sigma}$ is spherical. It follows easily that it must be either a (nonsingular) sphere, a football or a turnover with multiplicities $(2,2,2)$. (In fact $\widetilde{\Sigma}$ is a sphere because we assume $z\left(\pi_{1}^{\text {orb }}(\widetilde{\Sigma})\right)$ is trivial.) Hence the lemma.

Lemma 2.3 Let $Y$ denote an irreducible 3-orbifold with infinite $\pi_{1}^{\text {orb }}(Y)$. Then $z\left(\pi_{1}^{\text {orb }}(Y)\right)$ is torsion-free.

Proof By the JSJ-decomposition theorem for 3-orbifolds (see [5, Theorem 3.3]), there is a finite collection (possibly empty) of disjoint, essential toric 2-suborbifolds $\Sigma_{j}, j=1,2, \ldots, m$, which split $Y$ into 3 -suborbifolds $Y_{i}, i=1,2, \ldots, n$, such that each $Y_{i}$ is either Seifert fibered or atoroidal. This presents $\pi_{1}^{\text {orb }}(Y)$ as the fundamental group of a finite graph of groups, where the vertex groups are $\pi_{1}^{\text {orb }}\left(Y_{i}\right)$ and the edge groups are $\pi_{1}^{\mathrm{orb}}\left(\Sigma_{j}\right)$. If $\left\{\Sigma_{j}\right\}$ is not empty, then the torsion part of $z\left(\pi_{1}^{\mathrm{orb}}(Y)\right)$ must lie in the edge groups $z\left(\pi_{1}^{\text {orb }}\left(\Sigma_{j}\right)\right)$; see Lemma 2.1. By Lemma 2.2(a), $z\left(\pi_{1}^{\text {orb }}\left(\Sigma_{j}\right)\right)$ is torsion-free, which implies that $z\left(\pi_{1}^{\text {orb }}(Y)\right)$ is torsion-free when $\left\{\Sigma_{j}\right\}$ is not empty.

Suppose $\left\{\Sigma_{j}\right\}$ is empty. Then $Y$ is either Seifert fibered or atoroidal. Assume $Y$ is Seifert fibered first, and let $\pi: Y \rightarrow B$ be a Seifert fibration. There is an induced exact sequence (see [5, Proposition 2.12])

$$
1 \rightarrow C \rightarrow \pi_{1}^{\text {orb }}(Y) \stackrel{\pi_{*}}{\longrightarrow} \pi_{1}^{\text {orb }}(B) \rightarrow 1,
$$

where $C$ is cyclic or dihedral (either finite or infinite). In addition, $C$ is finite if and only if $\pi_{1}^{\text {orb }}(Y)$ is finite. In the present case $Y$ only has a 1 -dimensional singular set, so that a generic fiber of $\pi$ must be a circle. Consequently, $C$ is cyclic in the above exact sequence. Since $\pi_{1}^{\mathrm{orb}}(Y)$ is infinite, we have $C=\mathbb{Z}$. On the other hand, 
$C=\pi_{1}\left(\mathbb{S}^{1}\right) /$ Image $\delta$, where $\delta: \pi_{2}^{\text {orb }}(B) \rightarrow \pi_{1}\left(\mathbb{S}^{1}\right)$ is the connecting homomorphism in the exact sequence of homotopy groups associated to the Seifert fibration $\pi: Y \rightarrow B$. (For the definition of homotopy groups of orbifolds and the exact sequence associated to an orbifold fibration, see $[21 ; 22 ; 10]$.) As $C$ is infinite, $\delta$ must be the zero map, and consequently $\pi_{*}: \pi_{2}^{\text {orb }}(Y) \rightarrow \pi_{2}^{\text {orb }}(B)$ is surjective. By the assumption that $Y$ is irreducible, its universal cover $\tilde{Y}$ is also irreducible; see [5, Theorem 3.23]. Consequently, we have $\pi_{2}^{\text {orb }}(Y)=\pi_{2}(\tilde{Y})=0$ which implies that $\pi_{2}^{\text {orb }}(B)=0$. Now observing that a bad 2-orbifold and a spherical 2-orbifold must have nontrivial $\pi_{2}^{\text {orb }}$, we conclude, by Lemma 2.2 , that $z\left(\pi_{1}^{\text {orb }}(B)\right)$ must be torsion-free. It follows easily that $z\left(\pi_{1}^{\text {orb }}(Y)\right)$ is torsion-free in this case.

It remains to consider the case where $Y$ is atoroidal. If $Y$ is nonsingular (ie a 3manifold), then $\pi_{1}^{\text {orb }}(Y)=\pi_{1}(Y)$ is torsion-free; hence $z\left(\pi_{1}^{\text {orb }}(Y)\right)$ must be torsionfree. If $Y$ is an honest orbifold, then by the Orbifold theorem of Boileau, Leeb and Porti (see [4, Corollary 1.2]), $Y$ is geometric. In fact, we will need the following more precise statement: $Y$ has a metric of constant curvature or is Seifert fibered. It is clear that, since $\pi_{1}^{\text {orb }}(Y)$ is infinite, we only need to discuss the following two cases: (i) $Y$ is hyperbolic, (ii) $Y$ is Euclidean.

Suppose $Y$ is hyperbolic. Then there is a hyperbolic 3-manifold $Y^{\prime}$ and a finite group of isometries $G$ such that $Y=Y^{\prime} / G$. Now suppose $z\left(\pi_{1}^{\text {orb }}(Y)\right)$ is not torsion-free, and let $g \in z\left(\pi_{1}^{\mathrm{orb}}(Y)\right)$ be a torsion element. Then since $\pi_{1}\left(Y^{\prime}\right)$ is torsion-free, $g$ may be regarded as an element of $G$, and it acts on $Y^{\prime}$ via deck transformations. Moreover, $g$ must have a fixed point, say $p \in Y^{\prime}$. This gives rise to an automorphism $g_{*}$ of $\pi_{1}\left(Y^{\prime}, p\right)$, which is trivial because $g \in z\left(\pi_{1}^{\text {orb }}(Y)\right)$. By Mostow Rigidity, $g: Y^{\prime} \rightarrow Y^{\prime}$ is trivial, which is a contradiction.

Suppose $Y$ is Euclidean. By the Bieberbach theorem (see [41, page 443]), $Y$ is finitely covered by $T^{3}$ with deck transformation group $G$. Let $x \in z\left(\pi_{1}^{\text {orb }}(Y)\right)$ be a torsion element. Then $x$ may be regarded as an element of $G$ and acts on $T^{3}$ via deck transformations. Furthermore, $x$ must have a fixed point, say $p \in T^{3}$. Since $x$ is central, the induced automorphism $x_{*}: \pi_{1}\left(T^{3}, p\right) \rightarrow \pi_{1}\left(T^{3}, p\right)$ must be trivial. It follows that $x$ is trivial, which is a contradiction.

This completes the proof of the lemma.

Given any good 3-orbifold $Y$ which is not irreducible, one can cut $Y$ open along a finite system of spherical 2-suborbifolds into pieces which are irreducible. More precisely, by the spherical decomposition theorem (see [5, Theorem 3.2]), there is a finite, nonempty collection of disjoint spherical 2-suborbifolds $\left\{\Sigma_{j}\right\}$ such that each component $Y_{i}$ of $Y \backslash\left\{\Sigma_{j}\right\}$ becomes an irreducible 3-orbifold after capping-off the boundary spherical 2 -suborbifolds by the corresponding discal 3-orbifolds. 
For the purpose in this paper, a slightly improved version of the above statement is needed. More concretely, given any system of spherical 2 -suborbifolds $\left\{\Sigma_{j}\right\}$ of $Y$, let $\left\{Y_{i}\right\}$ be the set of components of $Y \backslash\left\{\Sigma_{j}\right\}$. We say that $\Sigma_{j}$ is separating (resp. nonseparating) in $Y_{i}$ if $\Sigma_{j}$ is a boundary component (resp. a nonseparating spherical 2-suborbifold) of the closure of $Y_{i}$ in $Y$. (Note that $\Sigma_{j}$ can be a nonseparating spherical 2-suborbifold of $Y$ but is separating in $Y_{i}$.) With this understood, we say that the corresponding spherical decomposition of $Y$ is reduced if for any $\Sigma_{j}, Y_{i}$ such that $\Sigma_{j}$ is separating in $Y_{i}, \pi_{1}^{\text {orb }}\left(\Sigma_{j}\right)$ is a proper subgroup of $\pi_{1}^{\text {orb }}\left(Y_{i}\right)$ under the inclusion of $\Sigma_{j}$ in the closure of $Y_{i}$ in $Y$.

Lemma 2.4 For any good, reducible 3-orbifold $Y$, there exists a reduced spherical decomposition of $Y$ into irreducible 3-orbifolds.

Proof Given any spherical decomposition of $Y$ into irreducible pieces, which always exists (see [5, Theorem 3.2]), we can modify it into a reduced spherical decomposition as follows. Let $\left\{\Sigma_{j}\right\}$ be the corresponding system of spherical 2-suborbifolds and let $\left\{Y_{i}\right\}$ be the set of components of $Y \backslash\left\{\Sigma_{j}\right\}$. Suppose for some $i, j, \Sigma_{j}$ is separating in $Y_{i}$ and $\pi_{1}^{\text {orb }}\left(\Sigma_{j}\right)=\pi_{1}^{\text {orb }}\left(Y_{i}\right)$. Let $Y_{k} \in\left\{Y_{i}\right\}$ be the other component whose closure in $Y$ also contains $\Sigma_{j}$ as a boundary component. Then observe that the 3-orbifold obtained from capping-off $Y_{k} \cup \Sigma_{j} \cup Y_{i}$ is the same as that obtained from capping-off $Y_{k}$. This is because, by the Geometrization theorem, the 3-orbifold obtained from capping-off the boundary components of $Y_{i}$ other than $\Sigma_{j}$ is a discal 3-orbifold with boundary $\Sigma_{j}$. Consequently, if we remove $\Sigma_{j}$ from $\left\{\Sigma_{j}\right\}$, the corresponding spherical decomposition still splits $Y$ into irreducible pieces. Continuing this process, we arrive at a reduced spherical decomposition in finitely many steps. Hence the lemma.

We remark that given any spherical decomposition of a good 3-orbifold $Y$, with $\left\{\Sigma_{j}\right\}$ being the system of spherical 2 -suborbifolds and $\left\{Y_{i}\right\}$ being the set of components of $Y \backslash\left\{\Sigma_{j}\right\}$, one has a corresponding finite graph of groups whose vertex groups and edge groups are given by $\left\{\pi_{1}^{\text {orb }}\left(Y_{i}\right)\right\}$ and $\left\{\pi_{1}^{\text {orb }}\left(\Sigma_{j}\right)\right\}$ respectively, such that $\pi_{1}^{\text {orb }}(Y)$ is naturally isomorphic to the fundamental group of the graph of groups. When the spherical decomposition is reduced, the corresponding graph of groups is also reduced in the sense that an edge group is always a proper subgroup of the vertex groups as long as the end points of the edge are distinct vertices. Given any finite graph of groups, one can always modify it into a reduced one without changing the isomorphism class of the fundamental groups by collapsing a number of edges. Lemma 2.4 is simply a manifestation of this principle in the geometric setting of spherical decomposition of 3 orbifolds. When there are no nonseparating spherical 2-suborbifolds, the existence and uniqueness of reduced spherical decompositions were proven in [37] (called efficient splittings therein). 
Next we give a classification of certain orientation-preserving finite group actions on $\mathbb{S}^{1} \times \mathbb{S}^{2}$. The case where the actions are free or have only isolated exceptional orbits was discussed in Meeks and Scott [32, Theorem 8.4]. Our discussion relies on the Equivariant sphere theorem of Meeks and Yau (see [33]) and Geometrization of finite group actions on $\mathbb{S}^{3}$ (compare also Dinkelbach and Leeb [14] via equivariant Ricci flow).

In order to state the result, we shall fix the following convention and notations. We orient $\mathbb{S}^{3}$ as the boundary of the unit ball in $\mathbb{C}^{2}$ and consider certain orientationpreserving $\mathbb{Z}_{2 m}$-actions on $\mathbb{S}^{3}$. When $m$ is even, there is only one such action up to a change of generators of $\mathbb{Z}_{2 m}$. When $m$ is odd, there are two nonequivalent such actions, and we shall denote the quotient orbifolds by $\mathbb{R P}_{m}^{3}$ and $\widetilde{R P}^{3}{ }_{m}$, respectively. More concretely, we fix a generator $t$ of $\mathbb{Z}_{2 m}$, and let

$$
\mathbb{R P}_{m}^{3}=\mathbb{S}^{3} / \mathbb{Z}_{2 m}, \quad \text { where } t \cdot\left(z_{1}, z_{2}\right)=\left(-z_{1}, \exp \left(\frac{\pi i}{m}\right) z_{2}\right)
$$

and

$$
\widetilde{\mathbb{R P}}_{m}{ }_{m}=\mathbb{S}^{3} / \mathbb{Z}_{2 m}, \quad \text { where } t \cdot\left(z_{1}, z_{2}\right)=\left(-z_{1}, \exp \left(\frac{(m+1) \pi i}{m}\right) z_{2}\right), m \text { is odd }
$$

Note that when $m>1$, these actions can be characterized by the fact that the whole group has no fixed points but the index 2 subgroup fixes an unknotted circle. Moreover, the difference between $\mathbb{R P}_{m}^{3}$ and $\widetilde{\mathbb{R P}^{3}}{ }_{m}$ is that the singular set of $\widetilde{R P}^{3}{ }_{m}$ has two components, of multiplicities 2 and $m$, respectively, while the singular set of $\mathbb{R P}_{m}^{3}$ has only one component, of multiplicity $m$.

Lemma 2.5 Let $G$ be a finite group that acts on $\mathbb{S}^{1} \times \mathbb{S}^{2}$ preserving the orientation.

- Suppose the action of $G$ is homologically trivial. Then $\mathbb{S}^{1} \times \mathbb{S}^{2} / G$ is the mapping torus of a periodic diffeomorphism of some spherical 2-orbifold.

- Suppose $G$ is cyclic and is generated by $t$ which is homologically nontrivial. Then the quotient orbifold $\mathbb{S}^{1} \times \mathbb{S}^{2} / G$ is diffeomorphic to one of the following:

$$
\mathbb{R P}_{m}^{3} \#_{m} \mathbb{R P}_{m}^{3}, \quad \mathbb{R P}_{m}^{3} \#_{m} \widetilde{\mathbb{R P}^{3}}{ }_{m}, \quad \text { or } \quad \widetilde{\mathbb{R P}^{3}}{ }_{m} \#_{m} \widetilde{\mathbb{R P}^{3}}{ }_{m},
$$

where $\#_{m}$ denotes the connected sum of orbifolds over a point of multiplicity $m$, such that a generator of the $\pi_{1}^{\text {orb }}$ of $\mathbb{S}^{2} / \mathbb{Z}_{m}$ has the same image on both sides of the connected sum.

Proof First of all, by the Equivariant sphere theorem of Meeks and Yau (see [33, page 480]), there exists a finite set of embedded 2-spheres $\left\{\Sigma_{i}\right\}$ of $\mathbb{S}^{1} \times \mathbb{S}^{2}$ that is $G$-invariant and generates the $\pi_{2}$ as a $\pi_{1}$-module. Since $\pi_{2}\left(\mathbb{S}^{1} \times \mathbb{S}^{2}\right)$ has rank 1 , 
we may assume $G$ acts on the set of spheres $\left\{\Sigma_{i}\right\}$ transitively. It follows easily from the Geometrization theorem that when cutting $\mathbb{S}^{1} \times \mathbb{S}^{2}$ open along the $\Sigma_{i}$ 's, each component $Y_{j}$ of $\mathbb{S}^{1} \times \mathbb{S}^{2} \backslash\left\{\Sigma_{i}\right\}$ is a 3-manifold diffeomorphic to the product of $\mathbb{S}^{2}$ with an interval.

For convenience of the argument, we shall consider the following finite graph $\Gamma$, where the vertices correspond to the components $Y_{j}$ and the edges to the embedded spheres $\Sigma_{i}$, and $\Sigma_{i}$ is incident to $Y_{j}$ if and only if $\Sigma_{i}$ is contained in the closure of $Y_{j}$. Clearly $\Gamma$ is homeomorphic to a circle, and there is an induced simplicial action of $G$ on $\Gamma$. We denote by $G_{0}$ the subgroup of $G$ which acts trivially on $\Gamma$.

Suppose $G_{0}$ is nontrivial. We pick an embedded sphere $\Sigma_{i}$ and cut $\mathbb{S}^{1} \times \mathbb{S}^{2}$ open along $\Sigma_{i}$. Because $\Sigma_{i}$ is $G_{0}$-invariant, we can close up $\mathbb{S}^{1} \times \mathbb{S}^{2} \backslash \Sigma_{i}$ and obtain a $G_{0}$-action on $\mathbb{S}^{3}$. By the Geometrization theorem, the action of $G_{0}$ is given by an isometry, which implies that the original $G_{0}$-action on $\mathbb{S}^{1} \times \mathbb{S}^{2}$ is a product action that is trivial on the $\mathbb{S}^{1}$-factor. Note that we are done if $G=G_{0}$.

Assume $G \neq G_{0}$ and consider the action of $G$. In the case where $G$ acts homologically trivially, $G / G_{0}$ acts effectively on $\Gamma$ by rotations. This implies that $\mathbb{S}^{1} \times \mathbb{S}^{2} / G$ is the mapping torus of the 2-orbifold $\mathbb{S}^{2} / G_{0}$ for some periodic diffeomorphism of $\mathbb{S}^{2} / G_{0}$ which generates $G / G_{0}$. The lemma follows easily in this case.

Suppose $G$ is generated by $t$ which is homologically nontrivial. Then the induced action of $t$ on $\Gamma$ must be given by a reflection, and $G_{0}$ is an index 2 subgroup. Furthermore, $G_{0}$ is cyclic in this case and the action of $G_{0}$ on $\mathbb{S}^{2}$ is given by rotations. The order of $t$ is even, say $2 m$, and there are two possibilities for the induced action of $t$ on the graph $\Gamma$ : (i) $t$ has an invariant edge, (ii) $t$ fixes two vertices.

In case (i), $t$ leaves an embedded sphere $\Sigma_{i}$ invariant (which is the only one because by assumption $G$ acts transitively on the set of spheres $\left\{\Sigma_{i}\right\}$ ). The induced action of $t$ on $\Sigma_{i}$ is orientation-reversing, and there are two nonequivalent actions when $m$ is odd. More concretely, if we identify $\Sigma_{i}$ with the unit sphere in $\mathbb{R}^{3}=\mathbb{R} \times \mathbb{C}$, then the actions are given by

$$
t \cdot(x, z)=\left(-x, \exp \left(\frac{\pi i}{m}\right) z\right), \quad \text { where }(x, z) \in \mathbb{R} \times \mathbb{C},
$$

and

$$
t \cdot(x, z)=\left(-x, \exp \left(\frac{(m+1) \pi i}{m}\right) z\right), \quad \text { where }(x, z) \in \mathbb{R} \times \mathbb{C}, m \text { is odd. }
$$

It follows easily that the quotient of a $t$-invariant regular neighborhood of $\Sigma_{i}$ is diffeomorphic to either $\mathbb{R P}_{m}^{3}$ or $\widetilde{\mathbb{R P}^{3}} m$ with a ball centered at a singular point of multiplicity $m$ removed. Moreover, the complement of the $t$-invariant regular neighborhood is a 
3-manifold $Y_{j}$ that is diffeomorphic to the product of $\mathbb{S}^{2}$ with an interval. The action of $t$ on $Y_{j}$ can be naturally extended to an $t$-action on $\mathbb{S}^{3}$ by capping-off the boundary of $Y_{j}$, which, by the Geometrization theorem, is equivalent to an isometry. Note that when $m>1, t^{2}$ has a 1 -dimensional fixed-point set. It follows easily that $Y_{j} /\langle t\rangle$ is also diffeomorphic to either $\mathbb{R P}_{m}^{3}$ or $\widetilde{R P}^{3} m$ with a ball centered at a singular point of multiplicity $m$ removed, and $\mathbb{S}^{1} \times \mathbb{S}^{2} / G$ is diffeomorphic to either $\mathbb{R P}_{m}^{3} \#_{m} \mathbb{R P}_{m}^{3}$, or $\mathbb{R P}_{m}^{3} \#_{m} \widetilde{\mathbb{R P}}_{m}{ }_{m}$, or $\widetilde{\mathbb{R P}}^{3}{ }_{m} \#_{m} \widetilde{\mathbb{R P}}_{m}{ }_{m}$ as claimed.

In case (ii) where $t$ fixes two vertices of the graph $\Gamma$, the set $\left\{\Sigma_{i}\right\}$ has two elements $\Sigma_{1}$ and $\Sigma_{2}$, and $\mathbb{S}^{1} \times \mathbb{S}^{2} \backslash\left\{\Sigma_{i}\right\}$ has two components $Y_{1}$ and $Y_{2}$, such that $Y_{1}$ and $Y_{2}$ are $t$-invariant and $t$ switches $\Sigma_{1}$ and $\Sigma_{2}$. Similarly, the $t$-actions on $Y_{1}$ and $Y_{2}$ can be extended to a $t$-action on $\mathbb{S}^{3}$ by capping-off the boundary, and by the Geometrization theorem, the quotients of $Y_{1}$ and $Y_{2}$ by $t$ are diffeomorphic to either $\mathbb{R P}_{m}^{3}$ or $\widetilde{\mathbb{R P}^{3}} m$, and the lemma follows in this case too.

We end with a lemma concerning existence of Seifert-type $T^{2}$-fibrations on a $4-$ manifold.

Lemma 2.6 Let $\pi: X \rightarrow Y$ be a principal $\mathbb{S}^{1}$-bundle over an orientable 3-orbifold where $Y$ is Seifert fibered. If the homotopy class of a regular fiber of the Seifert fibration on $Y$ lies in the image of $z\left(\pi_{1}(X)\right)$ under $\pi_{*}: \pi_{1}(X) \rightarrow \pi_{1}^{\text {orb }}(Y)$, then $\pi: X \rightarrow Y$ may be extended to a principal $T^{2}$-bundle over a 2 -orbifold.

Proof Let pr: $Y \rightarrow B$ be the Seifert fibration on $Y$ where $B$ is a $2-$ orbifold. (We note that $B$ must be orientable because the class of a regular fiber of pr lies in the center $z\left(\pi_{1}^{\text {orb }}(Y)\right)$.) Then the composition of $\pi$ with pr, $\Pi: X \rightarrow B$, defines $X$ as a $T^{2}$-bundle over $B$. We shall prove that $\Pi$ is principal, which is equivalent to the condition that $\Pi$ has a trivial monodromy representation.

To see that the monodromy representation of $\Pi$ is trivial, we consider an arbitrary loop $\gamma$ in $B$ lying in the complement of the singular set. Pick a base point $b_{0} \in \gamma$, and a base point $x_{0} \in \Pi^{-1}\left(b_{0}\right)$. Choose a section $\gamma^{\prime}$ of $\Pi$ over $\gamma$ through $x_{0}$, and a loop $\delta$ containing $x_{0}$ in $X$ that is a section of $\pi$ over the fiber of pr at $b_{0}$. Let $h$ be the fiber of $\pi$ containing $x_{0}$. With this understood, the monodromy representation of $\Pi$ is trivial if and only if the classes of $h, \delta$, and $\gamma^{\prime}$ in $\pi_{1}(X)$ commute. But this is clear because the classes of both $h$ and $\delta$ lie in the center $z\left(\pi_{1}(X)\right)$. Hence the lemma.

\section{Fiber-sum decomposition and fundamental group}

This section contains three subsections. Section 3.1 is devoted to a review of Bass-Serre theory and Rips-Zela theory, and it also contains a proof of Lemma 3.1 and Lemma 3.2. 
Section 3.2 is occupied by a proof of Theorem 1.5(1), as given through Lemma 3.3, Lemma 3.4, and Proposition 3.5. The last subsection, Section 3.3, contains the proof for Theorem 1.5(2).

\subsection{Some recollections in geometric group theory}

We begin with a brief review of the Bass-Serre theory of groups acting on trees, see eg $[13 ; 42]$ for more details.

Let $\Gamma$ be a connected, nonempty graph, with the set of vertices and edges denoted by $V \Gamma$ and $E \Gamma$, respectively, and the incidence functions denoted by $\iota, \tau: E \Gamma \rightarrow V \Gamma$. Recall that a group of graphs, denoted by $G_{\Gamma}$, consists of the following data: each $v \in V \Gamma$ and $e \in E \Gamma$ is assigned with a group $G(v)$ and $G(e)$, respectively, and for each $e \in E \Gamma$ there is a pair of boundary monomorphisms $\alpha: G(e) \rightarrow G(\iota e)$ and $\omega: G(e) \rightarrow G(\tau e)$.

Let $\Gamma_{0}$ be a maximal tree in $\Gamma$. The fundamental group of $G_{\Gamma}$ with respect to $\Gamma_{0}$, denoted by $\pi\left(G_{\Gamma}, \Gamma_{0}\right)$, is the group given by the following presentation:

- Generating set: $\left\{t_{e} \mid e \in E \Gamma\right\} \cup \bigcup_{v \in V \Gamma} G(v)$.

- Relations: the relations for $G(v), \forall v \in V \Gamma ; t_{e}^{-1} \alpha(g) t_{e}=\omega(g), \forall g \in G(e)$, $\forall e \in E \Gamma$; and $t_{e}=1, \forall e \in E \Gamma_{0}=E \Gamma \cap \Gamma_{0}$.

It is known that the isomorphism class of $\pi\left(G_{\Gamma}, \Gamma_{0}\right)$ is independent of $\Gamma_{0}$, and it is called the fundamental group of the graph of groups $G_{\Gamma}$.

Given any graph of groups $G_{\Gamma}$, there is a canonically constructed tree $T$, called the Bass-Serre tree, together with a canonical action of the fundamental group of $G_{\Gamma}$. Moreover, the graph of groups $G_{\Gamma}$ can be recovered from the action of its fundamental group on the Bass-Serre tree in a canonical way, which we describe below.

Let $G$ be a group acting on a tree $T$ without inversion, ie the action sends vertices to vertices and edges to edges such that every edge invariant under the action is being fixed. Let $\Gamma$ be the quotient graph, and $p: T \rightarrow \Gamma$ be the quotient map. Let $T^{\prime} \subset T$ be a subset and $T_{0} \subset T^{\prime}$ be a subtree of $T$. We call $T^{\prime}$ a fundamental $G$-transversal in $T$ with subtree $T_{0}$ if (i) $p: T^{\prime} \rightarrow \Gamma$ is bijective, and (ii) $p: T_{0} \rightarrow \Gamma$ is onto a maximal tree in $\Gamma$. It is known that such a pair $\left(T^{\prime}, T_{0}\right)$ always exists. Note that by (i), one can give a canonical graph structure to $T^{\prime}$ as follows: $V T^{\prime}=V T \cap T^{\prime}, E T^{\prime}=E T \cap T^{\prime}$, and the incidence functions $\bar{\imath}, \bar{\tau}: E T^{\prime} \rightarrow V T^{\prime}$ are defined by the equations

$$
p(\bar{\imath} e)=p(\iota e), p(\bar{\tau} e)=p(\tau e), \forall e \in E T^{\prime} .
$$


(Here $\iota, \tau$ are the incidence functions of $T$.) Note that by (ii), $T_{0}$ is a maximal tree in $T^{\prime}$ with respect to this graph structure, and $\bar{\imath} e=\iota e, \bar{\tau} e=\tau e$ for any $e \in E T_{0}$.

Now, given any fundamental $G$-transversal $T^{\prime}$ with subtree $T_{0}$, one can canonically construct a graph of groups $G_{\Gamma}$ as follows, where $\Gamma$ and $T^{\prime}$ are identified as graphs. For any $v \in V T^{\prime}$, we assign to it the group $G(v)=G_{v}=\{g \in G \mid g v=v\}$, and for any $e \in E T^{\prime}$, we assign to it the group $G(e)=G_{e}=\{g \in G \mid g e=e\}$. The boundary monomorphisms $\alpha: G(e) \rightarrow G(\bar{l} e), \omega: G(e) \rightarrow G(\bar{\tau} e)$ are defined as follows. For any $e \in E T^{\prime}$, pick $g_{e}, h_{e} \in G$ such that $g_{e} \bar{\imath} e=\iota e, h_{e} \bar{\tau} e=\tau e$, where for any $e \in E T_{0}$, $g_{e}=h_{e}=1$. Then for any $g \in G(e)$, define $\alpha(g)=g_{e}^{-1} g g_{e}$ and $\omega(g)=h_{e}^{-1} g h_{e}$ (note that $G(e) \subset G(\iota e), G(e) \subset G(\tau e)$ ).

There is an obvious homomorphism $\phi: \pi\left(G_{\Gamma}, T_{0}\right) \rightarrow G$ which sends $t_{e}$ to $g_{e}^{-1} h_{e} \in G$. The fundamental theorem of the Bass-Serre theory asserts that $\phi$ is an isomorphism. Moreover, when $T$ is the Bass-Serre tree of a graph of groups $G_{\Gamma}$ and $G$ is the fundamental group of $G_{\Gamma}$ with the canonical action on $T$, the graph of groups $G_{\Gamma}$ can be recovered in the above manner.

Next we review the Rips-Sela theory; see [38] for more details. Given any group $G$, a $Z$-splitting of $G$ is a presentation of $G$ as the fundamental group of a finite graph of groups where all the edge groups are infinite cyclic. Elementary $Z$-splittings are Z-splittings for which the graph of groups contains only one edge, ie an amalgamated product or an HNN extension. Given a Z-splitting of $G$ and an elementary Z-splitting of a vertex group of the $\mathrm{Z}$-splitting that is compatible with the boundary monomorphisms, there is a naturally defined new $\mathrm{Z}$-splitting of $G$, which is called an elementary refinement, where the new graph of groups is obtained by replacing the vertex in the original graph by the corresponding one edge graph. A refinement of a $\mathrm{Z}$-splitting is the result of a sequence of elementary refinements. The inverse operation of a refinement is called a collapse.

The fundamental result in the Rips-Sela theory concerns the existence of certain universal Z-splittings of a single-ended finitely presented group, called canonical JSJ decompositions, from which all other Z-splittings of the group can be derived in a certain organized way (involving refinement or collapse). The starting point of this work is an analysis of the interactions between two distinct elementary Z-splittings. To be more concrete, let $G=A_{i} *_{C_{i}} B_{i}$ (or $A_{i} * C_{i}$ ) be two given elementary Zsplittings, where $C_{i}$ is generated by $c_{i}$, for $i=1,2$. The element $c_{2}$ is called elliptic with respect to the first splitting if it is contained in a conjugate of $A_{1}$ or $B_{1}$, and hyperbolic otherwise, and similarly for $c_{1}$ with respect to the second splitting. With this understood, one of the basic result in the Rips-Sela theory (see [38, Theorem 2.1]) asserts that if $G$ is freely indecomposable, then $c_{1}$ and $c_{2}$ are simultaneously elliptic or simultaneously hyperbolic. 
The bulk of the Rips-Sela theory is devoted to the analysis of hyperbolic-hyperbolic splittings. Our first observation is that, for a group $G$ with infinite $z(G)$, hyperbolichyperbolic splittings seldom occur, which greatly simplifies the situation.

Lemma 3.1 Let $G$ be a single-ended group with infinite $z(G)$, and suppose $G$ is not isomorphic to the fundamental group of a 2-torus or Klein bottle. Then

(i) the center $z(G)$ is contained in the edge groups of every reduced $Z$-splitting of $G$, and

(ii) there are no hyperbolic-hyperbolic elementary $Z$-splittings of $G$.

Proof We shall first prove part (i) of the lemma, where it suffices to consider only the case of elementary Z-splittings. Let $G=A *_{C} B$ or $A *_{C}$ be an elementary Z-splitting, where $A \neq C \neq B$. By Lemma 2.1, if the splitting is an amalgamated product, then $C$ contains $z(G)$. If the splitting is an HNN extension and $C$ does not contain $z(G)$, then $A=C=\langle c\rangle$ which is infinite cyclic, and $G$ is isomorphic to $A *_{A} \alpha$ for a finite order automorphism $\alpha$ of $A$. Clearly $\alpha$ is either the identity or $\alpha: c \mapsto c^{-1}$, which implies that $G$ is isomorphic to the fundamental group of a 2-torus or Klein bottle. Hence part (i) of the lemma.

As for part (ii), suppose to the contrary, there is a pair of hyperbolic-hyperbolic elementary Z-splittings $G=A_{i} *_{C_{i}} B_{i}$ (or $A_{i} * C_{i}$ ), $i=1$, 2, where $C_{i}$ is generated by $c_{i}$. We first note that the hyperbolicity implies that the splittings are reduced. Then by part (i), there are integers $m, n>0$ such that $c_{1}^{m}, c_{2}^{n} \in z(G)$, so that $c_{1}^{m}$ and $c_{2}^{n}$ commute. With this understood, Theorem 3.6 in Rips and Sela [38] implies that $G$ is isomorphic to the fundamental group of either a 2-torus, or a Klein bottle, or a Euclidean 2-branched projective plane, or a Euclidean 4-branched sphere (an explicit presentation of these groups are given in Proposition 3.3 of [38], page 63). The case of 2-torus or Klein bottle is excluded by the assumptions of the lemma, and the rest of the cases are excluded by the fact that $G$ has infinite center; see Lemma 2.2. (Note that in Theorem 3.6 of [38], there is the assumption that $G$ is a freely indecomposable group which does not split over $\mathbb{Z}_{2}$. By Stallings' End theorem (see eg [42, Theorem 6.1]), $G$ satisfies this assumption because of being single-ended.) Hence the lemma.

We remark that hyperbolic-hyperbolic splittings do occur. For example, let $G$ be the fundamental group of a Klein bottle. Then $G=A *_{A} \alpha$, where $A=\langle c\rangle$ is infinite cyclic and $\alpha: c \mapsto c^{-1}$, and $G=A *_{C} A$, where $C$ is the index 2 subgroup of the infinite cyclic group $A$, are a pair of hyperbolic-hyperbolic splittings of $G$.

Let $G$ be a single-ended group with infinite $z(G)$, which is not isomorphic to the fundamental group of a 2-torus or Klein bottle. Let $T$ be the Bass-Serre tree of a 
reduced Z-splitting of $G$, and let $V$ be the subset of the set of vertices $V T$ which consists of $v$ such that the isotropy subgroup $G_{v}$ fixes a vertex $v^{\prime} \neq v$. The subset $V$ is clearly $G$-invariant, which gives rise to a $G$-invariant partition $(V, V T \backslash V)$ of $V T$. The following lemma is concerned with the structure of $V$.

Lemma 3.2 There exists a collection of infinite cyclic subgroups $G_{i}$ of $G, i \in I$, which has the following significance.

- For each $i \in I$, let $V_{i}$ be the subset of $V$ consisting of $v$ such that $G_{v}=G_{i}$, and let $H_{i} \equiv\left\{t \in G \mid \operatorname{tg} t^{-1}=g, \forall g \in G_{i}\right\}$ be the centralizer of $G_{i}$. Then $H_{i}$ acts transitively on $V_{i}$.

- For each $i \in I$, let $\left\{g_{j} \mid j \in J(i)\right\}$ be a fixed choice of representatives of the right cosets of $H_{i}$ in $G$, where the right coset $H_{i}$ is represented by $g_{j}=1$. Then $\left\{g_{j}\left(V_{i}\right) \mid j \in J(i), i \in I\right\}$ forms a partition of $V$.

Proof Let $v \in V$ be any element, and let $v^{\prime} \neq v$ be fixed under $G_{v}$. Since $T$ is a tree, there exists a unique reduced path $\gamma$ in $T$ which connects $v$ and $v^{\prime}$. Because $G_{v}$ fixes both $v$ and $v^{\prime}$, and because $\gamma$ is unique, $G_{v}$ must also fix $\gamma$. In particular, if $e$ is the edge in $\gamma$ which is incident to $v$, then it follows easily that $G_{v}=G_{e}$, which implies that $G_{v}$ is infinite cyclic.

Let $v_{1}$ be the other vertex in $\gamma$ to which $e$ is incident. Since the $\mathrm{Z}$-splitting is reduced, $v_{1}$ must lie in the same orbit of $v$ under the action of $G$. In other words, there is a $t \in G$ such that $t \cdot v=v_{1}$. Suppose $G_{v}=G_{e}$ is generated by $c$. Then $G_{v_{1}}=t G_{v} t^{-1}$ is generated by $c_{1} \equiv t c t^{-1}$. Furthermore, $c \in G_{e} \subset G_{v_{1}}$, so that $c=c_{1}^{n}$ for some $n \in \mathbb{Z}$. On the other hand, by Lemma 3.1(i), there exists a nonzero $m \in \mathbb{Z}$ such that $c^{m} \in z(G)$. Consequently,

$$
c_{1}^{m}=\left(t c t^{-1}\right)^{m}=t c^{m} t^{-1}=c^{m}=c_{1}^{n m},
$$

which implies $n=1$. With $c=c_{1}=t c t^{-1}$, it follows that $t$ lies in the centralizer of $G_{v}$, and moreover, $G_{v_{1}}=G_{v}$. Repeating this argument to $v_{1}$, we see that there is a $t^{\prime}$ lying in the centralizer of $G_{v}$, such that $t^{\prime} \cdot v=v^{\prime}$ and $G_{v^{\prime}}=G_{v}$. Now if we let $V(v)$ be the subset of $V$ consisting of elements whose isotropy subgroup equals $G_{v}$, and let $H(v)$ be the centralizer of $G_{v}$, then $H(v)$ acts transitively on $V(v)$.

The above analysis shows that the following relation $\sim$ on $V$ is an equivalence relation: $v^{\prime} \sim v$ if and only if $G_{v}$ fixes $v^{\prime}$. The equivalence relation gives rise to a partition of $V$. It is clear that one can choose a subset $\left\{V_{i} \mid i \in I\right\}$ of equivalence classes such that this partition can be described as $\left\{g_{j}\left(V_{i}\right) \mid j \in J(i), i \in I\right\}$, where $G_{i}$ is the isotropy subgroup of the vertices in $V_{i}$, and $g_{j}, j \in J(i)$, is some fixed representative of the right coset of the centralizer $H_{i}$ of $G_{i}$ in $G$, with $g_{j}=1$ for the right coset of $H_{i}$. This completes the proof of the lemma. 


\subsection{Proof of Theorem 1.5(1)}

By assumption, $X$ is fiber-sum-decomposed into $X_{i}$ along $N_{j}$. This gives rise to a Z-splitting of $\pi_{1}(X)$ which will be denoted by $\Lambda$, with vertex groups and edge groups given by $\pi_{1}\left(X_{i}\right)$ and $\pi_{1}\left(N_{j}\right)$, respectively. Note that Definition 1.3 (iv) implies that the Z-splitting $\Lambda$ is reduced. Furthermore, we shall point out that by Lemma 3.1(i), $z\left(\pi_{1}(X)\right)$ is contained in every edge group of $\Lambda$. On the other hand, recall that the fiber-sum decomposition of $X$ gives rise to a canonical injective $\mathbb{S}^{1}$-action on $X$. We denote the orbit map by $\pi: X \rightarrow Y$, where we shall point out that $Y$ is naturally a good orbifold, ie it does not contain any bad 2-suborbifolds. Let $\Sigma_{j}$ be the spherical 2-suborbifold of $Y$ over which $N_{j}$ is Seifert fibered under $\pi$. Then it follows easily that the decomposition of $Y$ in $Y_{i}$ along $\Sigma_{j}$ is a reduced spherical decomposition, where $Y_{i}$ is the irreducible 3 -orbifold in the orbit map $\pi_{i}: X_{i} \rightarrow Y_{i}$ that comes with the fiber-sum decomposition of $X$; see Definition 1.3.

Let $\Lambda_{J S J}$ be a canonical JSJ decomposition of $\pi_{1}(X)$ as constructed in [38]. We will show that $\Lambda_{J S J}$ and $\Lambda$ are equivalent as canonical JSJ decompositions of $\pi_{1}(X)$ as described in [38]. To this end, we consider the Bass-Serre trees $T_{J S J}$ and $T$ of $\Lambda_{J S J}$ and $\Lambda$, respectively, each equipped with the canonical action of $\pi_{1}(X)$. As for notations, recall that for any vertex $v$ or edge $e$ of $T_{J S J}$ or $T$, the corresponding isotropy subgroups of $\pi_{1}(X)$ are denoted by $G_{v}$ or $G_{e}$, respectively.

Lemma 3.3 For any $w \in V T, G_{w}$ fixes a vertex of $T_{J S J}$.

Proof We consider the induced action of $G_{w}$ on the Bass-Serre tree $T_{J S J}$, and for any vertex $v$ and edge $e$ of $T_{J S J}$, we denote by $G_{v}^{\prime}$ and $G_{e}^{\prime}$ the isotropy subgroups of the $G_{w}$-action at $v$ and $e$, respectively. By Theorem 4.12 in [13], there are the following three possibilities.

(a) $G_{w}$ fixes a vertex of $T_{J S J}$.

(b) There is a reduced infinite path, $v_{0}, e_{1}^{\epsilon_{1}}, v_{1}, e_{2}^{\epsilon_{2}}, \ldots$, in $T_{J S J}$ such that

$$
G_{v_{0}}^{\prime} \subset G_{v_{1}}^{\prime} \subset \cdots, G_{w}=\bigcup_{n \geq 0} G_{v_{n}}^{\prime}=\bigcup_{n \geq 1} G_{e_{n}}^{\prime}
$$

and for all $n \geq 1, G_{w} \neq G_{e_{n}}^{\prime}$.

(c) Some element of $G_{w}$ translates some edge $e$ of $T_{J S J}$, and for $C \equiv G_{e}^{\prime}$, either $G_{w}=B *_{C} D$ with $B \neq C \neq D$, or $G_{w}=B *_{C}$.

It remains to show that neither (b) nor (c) can occur. First, applying Lemma 3.1(i) to the $\pi_{1}(X)$-action on $T_{J S J}$, we see that $z\left(\pi_{1}(X)\right)$ fixes every edge of $T_{J S J}$. Secondly, 
note that there is a factor $X_{i}$ such that $G_{w}$ is conjugate to the subgroup $\pi_{1}\left(X_{i}\right)$ in $\pi_{1}(X)$. Finally, if $h$ denotes the homotopy class of a regular fiber of $\pi: X \rightarrow Y$, then $h \in z\left(\pi_{1}(X)\right) \cap G_{w}$, so that $h \in G_{e}^{\prime}$ for every edge $e$ of $T_{J S J}$.

With the preceding understood, we consider case (b) first. In this case, we have

$$
\pi_{1}^{\mathrm{orb}}\left(Y_{i}\right) \cong \pi_{1}\left(X_{i}\right) /\langle h\rangle \cong G_{w} /\langle h\rangle=\bigcup_{n \geq 1} G_{e_{n}}^{\prime} /\langle h\rangle=\bigcup_{n \geq 1} F_{n},
$$

where $F_{n}$ is a finite group, $F_{n} \subset F_{n+1}$, and $G_{w} /\langle h\rangle \neq F_{n}$ for all $n \geq 1$. Clearly, $\pi_{1}^{\text {orb }}\left(Y_{i}\right)$ can not be finite. To rule out the case where $\pi_{1}^{\text {orb }}\left(Y_{i}\right)$ is infinite, we note that $\pi_{1}^{\text {orb }}\left(Y_{i}\right)$ has a finite index torsion-free subgroup $H$ by the Geometrization theorem; see $[4 ; 31]$. Let $\tilde{H}$ be the corresponding subgroup of $G_{w} /\langle h\rangle$ under $\pi_{1}^{\text {orb }}\left(Y_{i}\right) \cong G_{w} /\langle h\rangle$. Then $\widetilde{H}=\bigcup_{n \geq 1} F_{n} \cap \widetilde{H}=\bigcup_{n \geq 1} \varnothing=\varnothing$, which is a contradiction. Hence case (b) is excluded.

For case (c), we set $C^{\prime}=C /\langle h\rangle, B^{\prime}=B /\langle h\rangle$, and $D^{\prime}=D /\langle h\rangle$; then

$$
G_{w} /\langle h\rangle=B^{\prime} *_{C^{\prime}} D^{\prime} \text {, with } B^{\prime} \neq C^{\prime} \neq D^{\prime} \text {, or } G_{w} /\langle h\rangle=B^{\prime} *_{C^{\prime}} .
$$

Since $C^{\prime}$ is a finite group, $G_{w} /\langle h\rangle$ has more than one end by Stallings' End theorem; see eg [42, Theorem 6.1]. However, since $Y_{i}$ is irreducible, the number of ends of $\pi_{1}^{\text {orb }}\left(Y_{i}\right)$ is at most 1 , which is a contradiction to $\pi_{1}^{\text {orb }}\left(Y_{i}\right) \cong G_{w} /\langle h\rangle$. This rules out case (c), and the lemma is proved.

Lemma 3.4 There exists a $\pi_{1}(X)$-equivariant bijection $\phi: V T \rightarrow V T_{J S J}$. In particular, for any $w \in V T, G_{w}=G_{\phi(w)}$.

Proof First, we let $V$ (resp. $V_{J S J}$ ) be the subset of $V T$ (resp. $V T_{J S J}$ ) described in Lemma 3.2, and let $G_{i}, V_{i}, H_{i}, g_{j}, j \in J(i), i \in I$, be as defined in Lemma 3.2 for $V T$.

Given any $w \in V T, G_{w}$ fixes a vertex $v \in V T_{J S J}$ by Lemma 3.3. On the other hand, since $\pi_{1}(X)$ has no hyperbolic-hyperbolic splittings (see Lemma 3.1(ii)), it follows from the construction of canonical JSJ decompositions in [38] that the action of $G_{v}$ on $T$ must also fix a vertex, say $w^{\prime}$. One has the obvious inclusion relations $G_{w} \subset G_{v} \subset G_{w^{\prime}}$. By Lemma 3.2, one always has $G_{w}=G_{w^{\prime}}$, so that $G_{v}=G_{w}$ must hold. We will discuss according to cases (i) $w \in V T \backslash V$ and (ii) $w \in V$.

In case (i), $w^{\prime}=w$. We claim that $v \in V T_{J S J} \backslash V_{J S J}$; in particular, $v$ is uniquely determined by $w$. To see this, suppose there is a $v_{1} \neq v$ such that $G_{v_{1}}=G_{v}$. Then by Lemma 3.2 there is a $t$ lying in the centralizer of $G_{v}$ such that $v_{1}=t \cdot v$. In particular, $t$ is not in $G_{v}=G_{w}$. This implies that $t \cdot w \neq w$, but $G_{t \cdot w}=G_{w}$, which is a contradiction to the assumption that $w \in V T \backslash V$. With this understood, we define 
$\phi$ from $V T \backslash V$ to $V T_{J S J} \backslash V_{J S J}$ by setting $\phi(w)=v$. It follows easily that $\phi$ is a $\pi_{1}(X)$-equivariant bijection between $T V \backslash V$ and $V T_{J S J} \backslash V_{J S J}$. (The surjectivity part uses the fact that for any vertex $v \in V T_{J S J}$, the action of $G_{v}$ on $T$ fixes a vertex. This is a consequence of Lemma 3.1(ii) by the construction of JSJ decompositions in [38].)

In case (ii) where $w \in V, v$ also lies in $V_{J S J}$ by a similar argument. We shall define $\phi: V \rightarrow V_{J S J}$ as follows. Let $V_{i, J S J}$ be the subset of $V_{J S J}$ consisting of vertices whose isotropy subgroups are given by $G_{i}$. Then for any fixed choice of $w_{i} \in V_{i}$, $v_{i} \in V_{i, J S J}$, there is an $H_{i}$-equivariant bijection $\phi: V_{i} \rightarrow V_{i, J S J}$ sending $w_{i}$ to $v_{i}$. Using the elements $g_{j}, j \in J(i)$, we can uniquely extend $\phi$ to a $\pi_{1}(X)$-equivariant bijection from $\bigcup_{j \in J(i)} g_{j}\left(V_{i}\right)$ to $\bigcup_{j \in J(i)} g_{j}\left(V_{i, J S J}\right)$, which defines $\phi$ from $V$ to $V_{J S J}$. This completes the proof of the lemma.

According to Rips and Sela [38, Theorem 7.1], canonical JSJ decompositions of a single-ended, finitely presented group $G$ are determined up to the following equivalence relation: the Bass-Serre trees are $G$-homotopy equivalent relative to the set of vertices. With this understood, Theorem 1.5(1) follows from part (1) of the following proposition. In (2)-(4) we list some consequences of (1) which will be used later in the proofs of Theorem 1.5(2), Theorem 1.1, and Theorem 1.2.

Proposition 3.5 (1) There exist subdivisions $T^{\prime}$ and $T_{J S J}^{\prime}$ of $T$ and $T_{J S J}$, respectively, and $\pi_{1}(X)$-equivariant simplicial maps $h_{1}: T^{\prime} \rightarrow T_{J S J}$ and $h_{2}: T_{J S J}^{\prime} \rightarrow T$ extending $\phi$ and $\phi^{-1}$, respectively ( $\phi$ as in Lemma 3.4), such that $h_{2} \circ h_{1}$ and $h_{1} \circ h_{2}$ are $\pi_{1}(X)$-homotopic, relative to the set of vertices, to the corresponding identity maps.

(2) There exists a bijection $\hat{\phi}: V \Lambda \rightarrow V \Lambda_{J S J}$, such that for any factor $X_{i}$ of the fiber-sum decomposition of $X, \pi_{1}\left(X_{i}\right)$ is conjugate in $\pi_{1}(X)$ to the vertex group at the vertex $\widehat{\phi}\left(X_{i}\right)$ of $\Lambda_{J S J}$. In particular, the number of factors $X_{i}$ and the conjugacy classes of subgroups $\pi_{1}\left(X_{i}\right)$ depend only on $\pi_{1}(X)$.

(3) The cardinality of $\left\{N_{j}\right\}$ depends only on $\pi_{1}(X)$.

(4) For any $N_{j}$, there is an edge $e_{j}$ of the graph of $\Lambda_{J S J}$ such that $\pi_{1}\left(N_{j}\right)$ is conjugate in $\pi_{1}(X)$ to the edge group at $e_{j}$, and vice versa. In particular, the set of conjugacy classes of subgroups $\pi_{1}\left(N_{j}\right)$ depends only on $\pi_{1}(X)$.

Proof Fixing a choice of $\phi$ in Lemma 3.4, we shall define the subdivision $T^{\prime}$ of $T$ and the simplicial map $h_{1}: T^{\prime} \rightarrow T$ as follows. For any edge $e \in E T$, there is a unique reduced path in $T_{J S J}$ which starts from $\phi(\iota e)$ and ends at $\phi(\tau e)$. There is a unique subdivision of $e$ such that $\phi$ can be extended to a simplicial map over 
$e$. Doing this to every edge of $T$, we obtain the subdivision $T^{\prime}$ and the simplicial map $h_{1}$. The whole construction is clearly $\pi_{1}(X)$-equivariant because $\phi$ is $\pi_{1}(X)$ equivariant and reduced paths with fixed ends in a tree are unique. The subdivision $T_{J S J}^{\prime}$ and the simplicial map $h_{2}$ are constructed similarly with $\phi$ replaced by $\phi^{-1}$. One can further subdivide $T^{\prime}$ (still denoted by $T^{\prime}$ for simplicity) so that $h_{1}$ can be regarded as a simplicial map to the subdivision $T_{J S J}^{\prime}$ of $T_{J S J}$. With this understood, $h_{2} \circ h_{1}: T^{\prime} \rightarrow T$ is $\pi_{1}(X)$-homotopic to the identity map relative to the set of vertices $V T$ because (i) it is identity on $V T$, and (ii) $T$ is a tree. The statement about $h_{1} \circ h_{2}$ follows similarly. This finishes the proof of part (1).

Part (2) is a direct consequence of Lemma 3.4. For part (3), recall that the set of edges of $\Lambda$ is identified with the set $\left\{N_{j}\right\}$. With this understood, observe that the underlying graphs of $\Lambda$ and $\Lambda_{J S J}$, which are given by $T / \pi_{1}(X)$ and $T_{J S J} / \pi_{1}(X)$, respectively, are homotopy equivalent, so that they have the same Euler characteristics. This shows that the Euler characteristic of $\Lambda$, ie the number of vertices minus the number of edges of $\Lambda$, depends only on $\pi_{1}(X)$. It follows that the cardinality of $\left\{N_{j}\right\}$ depends only on $\pi_{1}(X)$.

Finally, we give a proof for part (4). For any $N_{j}$, we choose an edge $e$ of $T$ whose $\pi_{1}(X)$-orbit corresponds to $N_{j}$. As we have shown in the proof of part (1), $h_{2} \circ h_{1}(e)$ is a path in $T$ which has the same initial and terminal points as $e$. Since $T$ is a tree, the loop formed by $h_{2} \circ h_{1}(e)$ and $e^{-1}$ must be reduced, which implies that $e$ lies in the image of $h_{2} \circ h_{1}(e)$. Let $e^{\prime}$ be an edge of $T_{J S J}$ lying in the path $h_{1}(e)$ such that $e$ is contained in the path $h_{2}\left(e^{\prime}\right)$. Then by the construction of $h_{1}, h_{2}$ in part (1), we have $G_{e} \subset G_{e^{\prime}} \subset G_{e}$, which implies that $G_{e}=G_{e^{\prime}}$. We name $e_{j}$ to be the edge of $\Lambda_{J S J}$ which corresponds to the $\pi_{1}(X)$-orbit of $e^{\prime}$. Then it follows that $\pi_{1}\left(N_{j}\right)$ is conjugate to the edge group of $\Lambda_{J S J}$ at $e_{j}$. Part (4) follows easily. This completes the proof of Proposition 3.5.

\subsection{Proof of Theorem $1.5(2)$}

Before turning to the proof of Theorem 1.5(2), we first give a geometric interpretation of the conjugacy classes of subgroups $\pi_{1}\left(N_{j}\right)$ in $\pi_{1}(X)$. We begin by observing that the submanifolds $N_{j}$ fall into two different types as follows. Let $\Gamma$ be the subgroup of $\pi_{1}(X)$ generated by the homotopy class of a regular fiber of $\pi: X \rightarrow Y$. Then $N_{j}$ falls into two cases according to (i) $\Gamma=\pi_{1}\left(N_{j}\right)$, or (ii) $\Gamma$ is a proper subgroup of $\pi_{1}\left(N_{j}\right)$. It is clear that case (i) corresponds to the case where $\Sigma_{j}$ is an ordinary $2-$ sphere.

With the preceding understood, we have the following lemma. 
Lemma 3.6 (1) Suppose $\Gamma$ is a proper subgroup of $\pi_{1}\left(N_{j}\right)$ for some $j$. Then for any $N_{k}$, if $g^{-1} \pi_{1}\left(N_{j}\right) g \subset \pi_{1}\left(N_{k}\right)$ for some $g \in \pi_{1}(X)$, then $g^{-1} \pi_{1}\left(N_{j}\right) g=$ $\pi_{1}\left(N_{k}\right)$. In particular, if $\pi_{1}\left(N_{j}\right)=z\left(\pi_{1}(X)\right)$, then $\pi_{1}\left(N_{k}\right)=z\left(\pi_{1}(X)\right)$ for any $k$.

(2) Let $N_{j}$ and $N_{k}$ be given which are over $\Sigma_{j}$ and $\Sigma_{k}$, respectively. Suppose there are components $\gamma_{j}$ and $\gamma_{k}$ of the singular set of $Y$ such that $\Sigma_{j} \cap \gamma_{j} \neq \varnothing$, $\Sigma_{k} \cap \gamma_{k} \neq \varnothing$, and suppose that $\pi_{1}\left(N_{j}\right)$ and $\pi_{1}\left(N_{k}\right)$ are conjugate in $\pi_{1}(X)$. Then $\gamma_{j}$ and $\gamma_{k}$ are equivalent in the following sense: either $\gamma_{j}=\gamma_{k}$, or there are components of the singular set of $Y, \gamma_{0}, \gamma_{1}, \ldots, \gamma_{N}$, and spherical 2-suborbifolds $\Sigma_{1}, \ldots, \Sigma_{N} \in\left\{\Sigma_{j}\right\}$, such that

$$
\gamma_{\alpha-1} \cap \Sigma_{\alpha} \cap \gamma_{\alpha} \neq \varnothing, \quad \alpha=1,2, \ldots, N .
$$

Proof For part (1), let $N_{j}$ and $N_{k}$ be Seifert fibered over $\Sigma_{j}$ and $\Sigma_{k}$, respectively, under $\pi: X \rightarrow Y$. Since $\Gamma$ is a proper subgroup of $\pi_{1}\left(N_{j}\right)$ and $g^{-1} \pi_{1}\left(N_{j}\right) g \subset \pi_{1}\left(N_{k}\right)$ for some $g \in \pi_{1}(X), \Gamma$ is also a proper subgroup of $\pi_{1}\left(N_{k}\right)$. Consequently, there are components $\gamma_{j}$ and $\gamma_{k}$ of the singular set of $Y$ such that $\Sigma_{j} \cap \gamma_{j} \neq \varnothing$ and $\Sigma_{k} \cap \gamma_{k} \neq \varnothing$. If $\gamma_{j}=\gamma_{k}$, one clearly has $g^{-1} \pi_{1}\left(N_{j}\right) g=\pi_{1}\left(N_{k}\right)$ as claimed.

Suppose $\gamma_{j} \neq \gamma_{k}$. We denote by $Y_{0}$ the 3 -orbifold obtained from $Y$ by removing a regular neighborhood of all singular circles of $Y$ except $\gamma_{k}$. Note that $Y_{0}$ is a good 3-orbifold as $Y$ is good. We let $\hat{Y}_{0}$ be a 3-manifold cover of $Y_{0}$. We shall apply the Equivariant loop theorem (see eg [5, Theorem 3.19]) to $\hat{Y}_{0}$ as follows. Denote by $F$ a component of $\partial \hat{Y}_{0}$ which contains the preimage of a meridian of $\gamma_{j}$. Then observe that the assumption $g^{-1} \pi_{1}\left(N_{j}\right) g \subset \pi_{1}\left(N_{k}\right)$ for some $g \in \pi_{1}(X)$ implies that $F$ is not $\pi_{1}-$ injective. Hence, by the Equivariant loop theorem, there is an equivariant compression 2-disc $\widehat{D}$ in $\hat{Y}_{0}$ with $\partial \hat{D} \subset F$. The group action on $\widehat{D}$ contains exactly one fixed point, which implies that the image of $\widehat{D}$ under the covering map $\hat{Y}_{0} \rightarrow Y_{0}$ is an embedded 2-disc $D$ in $\left|Y_{0}\right|$ intersecting $\gamma_{k}$ at exactly one point. Furthermore, it follows easily that $\partial D$ must be a meridian of $\gamma_{j}$. Closing up $D$ in $|Y|$, we obtain an embedded 2-sphere $\Sigma$, which intersects each of $\gamma_{j}$ and $\gamma_{k}$ at exactly one point and intersects no other singular circles. Since $Y$ contains no bad 2-suborbifolds, it follows that $\gamma_{j}$ and $\gamma_{k}$ must have the same multiplicity, which implies that $g^{-1} \pi_{1}\left(N_{j}\right) g=\pi_{1}\left(N_{k}\right)$ as claimed. If $\pi_{1}\left(N_{j}\right)=z\left(\pi_{1}(X)\right)$, then $\pi_{1}\left(N_{j}\right) \subset \pi_{1}\left(N_{k}\right)$ for any $k$ by Lemma 3.1(i), which implies that $\pi_{1}\left(N_{k}\right)=z\left(\pi_{1}(X)\right)$ for any $k$. This finishes off the proof of part (1).

Next we prove part (2). The idea is to show that up to replacing one or both of $\gamma_{j}$ and $\gamma_{k}$ by some singular circles that are equivalent in the sense described in part (2) of the lemma, the embedded $2-$ sphere $\Sigma$ which we constructed in the previous paragraph 
can be modified so that it lies in the complement of the spherical $2-$ suborbifolds $\left\{\Sigma_{j}\right\}$. To this end, we first perturb $\Sigma$ so that it intersects each element of $\left\{\Sigma_{j}\right\}$ transversely and the intersection occurs in the complement of the singular set of $Y$. Now we fix our attention on a $\Sigma^{\prime} \in\left\{\Sigma_{j}\right\}$ such that $\Sigma \cap \Sigma^{\prime} \neq \varnothing$. Let $l \in \Sigma \cap \Sigma^{\prime}$ be a circle (if there is any) which bounds a disc $D \subset \Sigma^{\prime}$ such that (i) $D$ contains no singular points, and (ii) $D$ contains no intersection points with $\Sigma$. Let $D_{1}$ and $D_{2}$ be the two discs into which $l$ divides $\Sigma$. Then both $D_{1} \cup D, D_{2} \cup D$ are embedded 2 -spheres in $Y$. Since $Y$ contains no bad 2-suborbifolds, it follows easily that exactly one of $D_{1}$ and $D_{2}$, say $D_{1}$, contains no singular points. With this understood, we shall modify $\Sigma$ by replacing $D_{1}$ with $D$ and slightly perturbing it by an isotopy so that the new surface does not intersect $\Sigma^{\prime}$ in a neighborhood of $D$. In order to keep the notation simple, we shall still denote the resulting embedded $2-$ sphere by $\Sigma$. It is easily seen that the above procedure has the effect of removing the component $l$ from $\Sigma \cap \Sigma^{\prime}$, and moreover, it does not create new intersection points of $\Sigma$ with any element of $\left\{\Sigma_{j}\right\}$. By repeating this procedure, we may assume now that the intersection of $\Sigma$ with any element $\Sigma^{\prime} \in\left\{\Sigma_{j}\right\}$ is either empty, or it consists of a union of circles each of which divides $\Sigma^{\prime}$ into two discs, each containing exactly one singular point.

One can further reduce the number of components of $\Sigma \cap \Sigma^{\prime}$ to at most one. To see this, let $l$ and $l^{\prime}$ be a pair of components of $\Sigma \cap \Sigma^{\prime}$ such that $l$ and $l^{\prime}$ bound an annulus $A^{\prime} \subset \Sigma^{\prime}$ and $l$ bounds a disc $D^{\prime} \subset \Sigma^{\prime}$ where $A^{\prime}$ and $D^{\prime}$ do not contain any intersection points with $\Sigma$ (note that if the number of components of $\Sigma \cap \Sigma^{\prime}$ is greater than 1 , such a pair always exists). Then the annulus $A \subset \Sigma$ bounded by $l$ and $l^{\prime}$ does not contain any singular points, because otherwise, either $l$ or $l^{\prime}$, say $l$, will bound a disc $D \subset \Sigma$ containing no singular points, and furthermore, $D$ and a disc in $\Sigma^{\prime}$ bounded by $l$ form an embedded 2 -sphere in $Y$ containing exactly one singular point, contradicting the fact that $Y$ is pseudogood. With this understood, we modify $\Sigma$ by replacing the annulus $A$ with $A^{\prime}$, and as before, after applying a small isotopy the pair of components $l$ and $l^{\prime}$ are removed and no new intersection points are created. By repeating this procedure, we may assume that for each $\Sigma^{\prime} \in\left\{\Sigma_{j}\right\}$, the intersection $\Sigma \cap \Sigma^{\prime}$ consists of at most one component.

Now we are at the final stage of modifying $\Sigma$. Let $l$ be a circle of intersection of $\Sigma$ with a $\Sigma^{\prime} \in\left\{\Sigma_{j}\right\}$ such that $l$ bounds a disc $D \subset \Sigma$ which does not intersect with any other elements of $\left\{\Sigma_{j}\right\}$. (Such $l$ always exists, or $\Sigma$ lies in the complement of $\left\{\Sigma_{j}\right\}$.) Let $D^{\prime} \subset \Sigma^{\prime}$ be a disc bounded by $l$. Then $D \cup D^{\prime}$ is an embedded 2-sphere which can be perturbed so that it lies in the complement of $\left\{\Sigma_{j}\right\}$. Call it $\widehat{\Sigma}$, and suppose that $\widehat{\Sigma}$ lies in $Y_{i}$, which is an irreducible 3 -orbifold. Furthermore, without loss of generality we assume $D$ contains a singular point in $\gamma_{j}$, and we denote by $\gamma_{j}^{\prime}$ the singular circle which intersects with $D^{\prime}$. We claim that $\gamma_{j}$ and $\gamma_{j}^{\prime}$ are equivalent in 
the sense described in part (2) of the lemma. To see this, note that $\hat{\Sigma}$ bounds a discal 3-orbifold in $Y_{i}$ by the irreducibility of $Y_{i}$. In particular, there is an arc $\gamma$ lying in the singular set of $Y_{i}$ which connects the two singular points on $\hat{\Sigma}$. If $\gamma$ does not intersect any elements of $\left\{\Sigma_{j}\right\}$, then $\gamma_{j}=\gamma_{j}^{\prime}$; hence they are equivalent. Suppose $\Sigma_{1}, \ldots, \Sigma_{N}$ are the elements of $\left\{\Sigma_{j}\right\}$ that intersect with $\gamma$. Then there are subarcs $I_{1}, \ldots, I_{N}$ of $\gamma$, where $I_{\alpha}$ is contained in the discal 3-orbifold in $Y_{i}$ bounded by $\Sigma_{\alpha}$, $1 \leq \alpha \leq N$. Clearly there are singular circles $\gamma_{0}, \gamma_{1}, \ldots, \gamma_{N}$ such that the end points of $I_{\alpha}$ lie in $\gamma_{\alpha-1}$ and $\gamma_{\alpha}$, respectively. It follows easily that $\gamma_{j}$ and $\gamma_{j}^{\prime}$ are equivalent through $\gamma_{0}, \ldots, \gamma_{N}$ and $\Sigma_{1}, \ldots, \Sigma_{N}$. With this understood, we replace $\gamma_{j}$ by $\gamma_{j}^{\prime}$, and we modify $\Sigma$ by replacing $D$ by $D^{\prime}$. The new embedded 2 -sphere can be perturbed slightly so that it does not intersect $\Sigma^{\prime}$ and no new intersection points with elements of $\left\{\Sigma_{j}\right\}$ were created. Furthermore, it intersects with each of the singular circles $\gamma_{k}$ and $\gamma_{j}^{\prime}$ in exactly one point and contains no other singular points. By repeating this procedure, we obtain an embedded $2-$ sphere, which is still denoted by $\Sigma$, such that (i) $\Sigma$ is in the complement of the elements of $\left\{\Sigma_{j}\right\}$, and (ii) $\Sigma$ contains exactly two singular points lying on some singular components $\widehat{\gamma}_{j}$ and $\hat{\gamma}_{k}$, which are equivalent to $\gamma_{j}$ and $\gamma_{k}$, respectively. As we have shown earlier, $\hat{\gamma}_{j}$ and $\hat{\gamma}_{k}$ are equivalent, which implies that $\gamma_{j}$ and $\gamma_{k}$ are equivalent. This finishes the proof of the lemma.

In summary, the conjugacy classes of subgroups $\pi_{1}\left(N_{j}\right)$ (which are the conjugacy classes of the edge groups of $\Lambda$ ) can be classified as follows: (i) there is a distinguished conjugacy class, ie the class of those $\pi_{1}\left(N_{j}\right)=\Gamma$, and this conjugacy class can be characterized by the fact that the corresponding $\Sigma_{j}$ are ordinary $2-$ spheres; (ii) for any other conjugacy class where $\pi_{1}\left(N_{j}\right)$ contains $\Gamma$ as a proper subgroup, there is an associated equivalence class of singular circles as described in Lemma 3.6(2), which is characterized by the fact that $\pi_{1}\left(N_{j}\right)$ belongs to the conjugacy class if and only if the corresponding $\Sigma_{j}$ intersects with a singular circle belonging to the equivalence class. With this understood, we shall show in the next lemma that, by modifying the embeddings of $N_{j}$ via fiber-preserving isotopies (with respect to $\pi: X \rightarrow Y$ ) if necessary, one can bring the underlying graph of the Z-splitting $\Lambda$ into a certain normal form. We should point out that modifying the embeddings of $N_{j}$ via fiber-preserving isotopies does not change the conjugacy classes of the edge groups of the Z-splitting.

Lemma 3.7 For any given vertex $v$ of $\Lambda$, and any conjugacy class of edge groups of $\Lambda$ that are contained in the vertex group $G(v)$ up to conjugacy, one can modify the embeddings of those $N_{j}$ via fiber-preserving isotopies, where $\pi_{1}\left(N_{j}\right)$ belongs to the given conjugacy class of edge groups, such that the $Z$-splitting of $\pi_{1}(X)$ associated to the new fiber-sum decomposition of $X$ has the following property: for any edge $e$, if $G(e)$ belongs to the given conjugacy class of edge groups, then $e$ is incident to $v$. 
Proof First of all, we observe that modifying the embeddings of $N_{j}$ via fiber-preserving isotopies corresponds to moving one of the points $y_{j, 1}$ or $y_{j, 2}$ (see Definition 1.3) via isotopies, and moreover, for any $Y_{i}$, the edge which corresponds to $N_{j}$ is incident to the vertex corresponding to $X_{i}$ if and only if one of the points $y_{j, 1}$ or $y_{j, 2}$ lies in $Y_{i}$.

Now with the vertex $v$ and the conjugacy class of edge groups given as in the lemma, we denote by $X_{0}$ the irreducible $\mathbb{S}^{1}$-four-manifold corresponding to $v$, and denote by $Y_{0}$ the corresponding irreducible 3 -orbifold. We first note that the case where the given conjugacy class of edge groups is the distinguished one, ie where $\pi_{1}\left(N_{j}\right)=\Gamma$, is trivial, because in this case $\Sigma_{j}$ is an ordinary 2 -sphere and hence the points $y_{j, 1}$ and $y_{j, 2}$ are both lying in the complement of the singular set. For any other conjugacy class of edge groups, there is an associated equivalence class of singular circles as described in Lemma 3.6(2). Since the edge groups belonging to the given conjugacy class are contained in the vertex group $G(v)=\pi_{1}\left(X_{0}\right)$ up to conjugacy, there must be a singular circle belonging to the equivalence class which has nonempty intersection with the irreducible 3-orbifold $Y_{0}$. We pick one such singular circle and denote it by $\gamma_{0}$, and we set $I_{0} \equiv Y_{0} \cap \gamma_{0} \neq \varnothing$. Now consider any $N_{j}$ such that $\pi_{1}\left(N_{j}\right)$ belongs to the given conjugacy class of edge groups and $\Sigma_{j} \cap \gamma_{0} \neq \varnothing$. There are two possibilities: (i) $\Sigma_{j}$ intersects $\gamma_{0}$ at two points; (ii) $\Sigma_{j}$ intersects $\gamma_{0}$ at only one point. Consider case (i) first. If we cut $Y$ open along $\Sigma_{j}$ and then fill in the 3-discal neighborhoods of $y_{j, 1}$ and $y_{j, 2}$, the singular circle $\gamma_{0}$ is turned into two components, one of which, denoted by $\gamma^{\prime}$, contains $I_{0}$. Without loss of generality, assume $y_{j, 1}$ is contained in $\gamma^{\prime}$. Then by moving $y_{j, 1}$ along $\gamma^{\prime}$ via isotopy if necessary, we may arrange such that $y_{j, 1} \in I_{0}$. Now consider case (ii). Let $\gamma_{1}$ be the singular circle which contains the other singular point on $\Sigma_{j}$. Then when we cut $Y$ open along $\Sigma_{j}$ and fill in the 3-discal neighborhoods of $y_{j, 1}$ and $y_{j, 2}$, the two components $\gamma_{0}$ and $\gamma_{1}$ are turned into one component, denoted by $\gamma^{\prime}$. In this case, one can always arrange so that $y_{j, 1} \in I_{0}$, by moving $y_{j, 1}$ via isotopy along $\gamma^{\prime}$. Note that after moving $y_{j, 1}$ via isotopy and then performing the connected sum operation to get back to $Y$, the singular circles $\gamma_{0}$ and $\gamma_{1}$ are turned into $\gamma_{0}^{\prime}$ and $\gamma_{1}^{\prime}$, respectively, both of which have nonempty intersection with $Y_{0}$. With this last property understood, observe that we can now perform the operation described above to any $N_{j}$ such that $\Sigma_{j} \cap \gamma_{1}^{\prime} \neq \varnothing$. The lemma follows by an induction process.

We remark that applying Lemma 3.7 to a $\mathrm{Z}$-splitting $\Lambda$ does not change the sets $V \Lambda$ and $E \Lambda$; it only changes the incident function. From the construction of Bass-Serre trees (see [13]), it follows particularly that neither the action of $\pi_{1}(X)$ on the vertex set of the Bass-Serre tree $T$ of $\Lambda$ changes, nor does the $\pi_{1}(X)$-equivariant bijection $\phi$ in Lemma 3.4. 
Proof of Theorem 1.5(2) First of all, we shall reformulate the problem as follows. We denote the group $\pi_{1}\left(X^{\prime}\right)$ by $G$ and identify $\pi_{1}(X)$ with $G$ via the given isomorphism $\alpha: \pi_{1}(X) \rightarrow \pi_{1}\left(X^{\prime}\right)$. With this understood, let $\Lambda$ and $\Lambda^{\prime}$ be the Z-splittings of $G$ associated to the given fiber-sum decompositions of $X$ and $X^{\prime}$, respectively. We shall prove that after modifying the embeddings of $N_{j}$ and $N_{j}^{\prime}$ via fiber-preserving isotopies if necessary, $\Lambda$ and $\Lambda^{\prime}$ may be arranged to be isomorphic as Z-splittings of $G$. Note that the assumption that $N_{j}$ and $N_{j}^{\prime}$ are null-homologous is equivalent to that the underlying graphs of $\Lambda$ and $\Lambda^{\prime}$ are trees. We shall denote by $T$ and $T^{\prime}$ the Bass-Serre trees of $\Lambda$ and $\Lambda^{\prime}$, respectively. By Lemma 3.4, there exists a $G$-equivariant bijection $\phi$ from $V T$ onto $V T^{\prime}$, which induces a bijection $\hat{\phi}: V \Lambda \rightarrow V \Lambda^{\prime}$ and a family of isomorphisms of vertex groups $\rho_{v}: G(v) \rightarrow G\left(v^{\prime}\right)$ given by conjugation by elements of $G$, where $v \in V \Lambda$ and $v^{\prime}=\widehat{\phi}(v) \in V \Lambda^{\prime}$.

First consider the special case where $\pi_{1}\left(N_{j}\right)=z(G)=\pi_{1}\left(N_{j}^{\prime}\right)$ for all $N_{j}$ and $N_{j}^{\prime}$. We fix a vertex $v \in V \Lambda$ and let $v^{\prime}=\widehat{\phi}(v) \in V \Lambda^{\prime}$ be the corresponding vertex. Then we apply Lemma 3.7 to $\Lambda$ and $\Lambda^{\prime}$ so that for the resulting new Z-splittings, which are still denoted by $\Lambda$ and $\Lambda^{\prime}$ for simplicity, every edge $e \in E \Lambda$ and $e^{\prime} \in E \Lambda^{\prime}$ is incident to $v$ and $v^{\prime}$, respectively. With this understood, there is an isomorphism of the underlying graphs of $\Lambda$ and $\Lambda^{\prime}$, extending $\hat{\phi}: V \Lambda \rightarrow V \Lambda^{\prime}$. Since by assumption all the edge groups of $\Lambda$ and $\Lambda^{\prime}$ are given by the center $z(G)$, it follows easily that the family of isomorphisms $\rho_{v}$ can be extended to an isomorphism of the Z-splittings $\Lambda$ and $\Lambda^{\prime}$. This finishes the proof for the special case where $\pi_{1}\left(N_{j}\right)=z(G)=\pi_{1}\left(N_{j}^{\prime}\right)$ for all $N_{j}$ and $N_{j}^{\prime}$.

Suppose $\pi_{1}\left(N_{j}\right)=z(G)$ for all $N_{j}$ does not hold. Then by Lemma 3.6(1), the condition that $\pi_{1}$ of a regular fiber of $\pi: X \rightarrow Y$ is a proper subgroup of $\pi_{1}\left(N_{j}\right)$ for some $N_{j}$ is equivalent to the more convenient condition that $\pi_{1}\left(N_{j}\right) \neq z(G)$, as the latter is formulated without reference to $\pi: X \rightarrow Y$. On the other hand, by Proposition 3.5(4), $\pi_{1}\left(N_{j}^{\prime}\right)=z(G)$ for all $N_{j}^{\prime}$ also does not hold. Accordingly, one can divide the set of edges $E \Lambda$ (resp. $E \Lambda^{\prime}$ ) into two groups by the following rules:

(I) $e \in E \Lambda$ (resp. $e^{\prime} \in E \Lambda^{\prime}$ ) belongs to (I) if and only if $G(e) \neq z(G)$ (resp. $\left.G\left(e^{\prime}\right) \neq z(G)\right)$.

(II) $e \in E \Lambda$ (resp. $e^{\prime} \in E \Lambda^{\prime}$ ) belongs to (II) if and only if $G(e)=z(G)$ (resp. $\left.G\left(e^{\prime}\right)=z(G)\right)$.

Pick a vertex $v \in V \Lambda$, and without loss of generality, assume that there is an edge $e$ belonging to (I) such that $G(e)$ is conjugate to a subgroup of $G(v)$. We denote the set of such edges by $E_{v}$. Then by Lemma 3.7, we can assume that any $e \in E_{v}$ is incident to $v$. Furthermore, we can assume (again with the help of Lemma 3.7) that 
any $e \in E \Lambda$ belonging to (II) is not incident to $v$ by the fact that $E_{v} \neq \varnothing$. With this understood, we denote by $\Gamma_{v}$ the minimal subgraph containing $v$ and $E_{v}$ and by $G_{\Gamma_{v}}$ the corresponding subgraph of groups supported by $\Gamma_{v}$. Finally, we let $v^{\prime}=\widehat{\phi}(v) \in V \Lambda^{\prime}$ be the corresponding vertex in the Z-splitting $\Lambda^{\prime}$. We make the same arrangement as above for the vertex $v^{\prime}$ with the corresponding notations in which $v$ is replaced by $v^{\prime}$.

Our next goal is to construct an isomorphism between the subgraphs of groups $G_{\Gamma_{v}}$ and $G_{\Gamma_{v^{\prime}}}$, extending the given isomorphism $\rho_{v}: G(v) \rightarrow G\left(v^{\prime}\right)$. To this end, we pick a fundamental $G$-transversal for $G_{\Gamma_{v}}$ as follows. Let $\tilde{v}$ be a vertex of the Bass-Serre tree $T$ whose $G$-orbit is $v$. For each $e \in E_{v}$, we choose an edge $\tilde{e} \in E T$ incident to $\tilde{v}$, whose $G$-orbit is $e$. We let $\Gamma_{\tilde{v}}$ be the minimal subgraph of $T$ containing $\tilde{v}$ and $\tilde{e}$, $\forall e \in E_{v}$. Then it is clear that $\Gamma_{\tilde{v}}$ is a fundamental $G$-transversal for $G_{\Gamma_{v}}$. With this understood, we shall construct a fundamental $G$-transversal for $G_{\Gamma_{v^{\prime}}}$ as follows.

We set $\tilde{v}^{\prime}=\phi(\tilde{v})$, where $\phi: V T \rightarrow V T^{\prime}$ is the $G$-equivariant bijection coming from Lemma 3.4, which induces $\widehat{\phi}: V \Lambda \rightarrow V \Lambda^{\prime}$. For any edge $\tilde{e} \in \Gamma_{\tilde{v}}$, we denote by $\tilde{w}$ the vertex other than $\tilde{v}$ to which $\tilde{e}$ is incident, and set $\tilde{w}^{\prime}=\phi(\tilde{w})$ correspondingly. Then as in the proof of Proposition 3.5, there exists a unique reduced path in $T^{\prime}$ connecting $\tilde{v}^{\prime}$ to $\tilde{w}^{\prime}$,

$$
v_{0}=\tilde{v}^{\prime}, \quad e_{1}^{\epsilon_{1}}, \quad v_{1}, \quad e_{2}^{\epsilon_{2}}, \quad \ldots, \quad e_{n}^{\epsilon_{n}}, \quad v_{n}=\tilde{w}^{\prime},
$$

such that $G_{\tilde{e}} \subset G_{e_{i}}$ for all $i$ and that there exists a $j$ with $G_{e_{j}}=G_{\tilde{e}}$. Let $\widehat{e}_{i} \in E \Lambda^{\prime}$ be the $G$-orbit of $e_{i}$. Then since the edge $e \in E_{v}$ belongs to (I), it follows that $\hat{e}_{j} \in E \Lambda^{\prime}$ also belongs to (I) because $G_{e_{j}}=G_{\tilde{e}}$. Now with $G_{e_{j}}=G_{\tilde{e}} \subset G_{e_{i}}$, it follows from Lemma 3.6(1) that $G_{e_{j}}=G_{e_{i}}$ for all $i$, which implies that the edge groups $G\left(\hat{e}_{i}\right)$ belong to the same conjugacy class in $G$. It follows that the vertices $v_{k}$, where $k$ is even, must be in the same $G$-orbit, and that $n$ must be odd. In particular, $v_{n-1}$ and $v_{0}=\tilde{v}^{\prime}$ are in the same $G$-orbit. We fix a choice of $g_{\tilde{e}} \in G$ such that $g_{\tilde{e}} v_{n-1}=\tilde{v}^{\prime}$, set $\tilde{e}^{\prime}=e_{n}$, and let $w \in V \Lambda$ and $w^{\prime} \in V \Lambda^{\prime}$ be the $G$-orbit of $\tilde{w}$ and $\tilde{w}^{\prime}$, respectively. Then the $G$-orbit $e^{\prime} \in E \Lambda^{\prime}$ of $\tilde{e}^{\prime}$ is incident to the vertices $v^{\prime}$ and $w^{\prime}$. It follows that $e^{\prime}, w^{\prime}$ are part of the subgraph $\Gamma_{v^{\prime}}$, and $v \mapsto v^{\prime}, e \mapsto e^{\prime}$ and $w \mapsto w^{\prime}$ define an isomorphism between $\Gamma_{v}$ and $\Gamma_{v^{\prime}}$.

Suppose $\rho_{v}: G(v) \rightarrow G\left(v^{\prime}\right)$ is given by $h \mapsto g_{\tilde{v}} h g_{\tilde{v}}^{-1}$ for some $g_{\tilde{v}} \in G$, where $h \in G_{\tilde{v}}$. Then the subset $\left\{g_{\tilde{v}} \tilde{v}^{\prime}, g_{\tilde{v}} g_{\tilde{e}} \tilde{e}^{\prime}, g_{\tilde{v}} g_{\tilde{e}} \tilde{w}^{\prime} \mid e \in E_{v}, w \in \Gamma_{v}\right\}$ is a fundamental $G-$ transversal for $G_{\Gamma_{v^{\prime}}}$. Moreover, there is an isomorphism $\left\{\rho_{v}, \rho_{e}, \rho_{w} \mid e \in E_{v}, w \in \Gamma_{v}\right\}$ between the subgraphs of groups $G_{\Gamma_{v}}$ and $G_{\Gamma_{v^{\prime}}}$, extending the given isomorphism $\rho_{v}: G(v) \rightarrow G\left(v^{\prime}\right)$, where $\rho_{e}: G_{\tilde{e}} \rightarrow G_{g_{\tilde{v}} g_{\tilde{e}} \tilde{e}^{\prime}}$ and $\rho_{w}: G_{\tilde{w}} \rightarrow G_{g_{\tilde{v}} g_{\tilde{e}} \tilde{w}^{\prime}}$ are given by conjugation of $g_{\tilde{v}} g_{\tilde{e}} \in G$.

Finally, by repeating the above construction, we obtain a disjoint union of subgraphs of groups $G_{\Gamma_{k}}$ of the Z-splitting $\Lambda$, a disjoint union of subgraphs of groups $G_{\Gamma_{k}^{\prime}}$ of the 
Z-splitting $\Lambda^{\prime}$, and a collection of isomorphisms $\rho_{k}: G_{\Gamma_{k}} \rightarrow G_{\Gamma_{k}^{\prime}}$, such that for any edges $e \in E \Lambda \backslash\left\{\Gamma_{k}\right\}$ and $e^{\prime} \in E \Lambda^{\prime} \backslash\left\{\Gamma_{k}^{\prime}\right\}, G(e)=z(G)=G\left(e^{\prime}\right)$. It follows easily that the isomorphisms $\rho_{k}$ can be uniquely extended to an isomorphism of Z-splittings between $\Lambda$ and $\Lambda^{\prime}$. This finishes the proof of Theorem 1.5(2).

\section{Irreducible $\mathbb{S}^{1}$-four-manifolds}

This section is devoted to a proof of Theorem 1.6. The proof involves a smooth classification of fixed-point free, smooth $\mathbb{S}^{1}$-four-manifolds whose $\pi_{1}$ has a center of rank greater than 1 (see Theorem 4.3), which is given at the end of the section.

The following lemma shows that a finitely generated group with infinite center is either single-ended or double-ended.

Lemma 4.1 Let $G$ be a finitely generated group with infinite $z(G)$ and suppose $G$ is not single-ended. Then $G$ is isomorphic to $A *_{A} \alpha$, where $A$ is a finite group. In particular, $G$ is double-ended.

Proof Let $e(G)$ denote the number of ends of $G$. Then $e(G) \geq 1$ because $G$ is infinite. On the other hand, by Stallings' End theorem (see eg Scott and Wall [42]), if $e(G) \geq 2$, then $G$ splits over a finite subgroup, ie either $G=A *_{C} B$ with $A \neq C \neq B$, or $G=A *_{C} \alpha$, where in both cases $C$ is a finite group. By Lemma 2.1, the assumption that $z(G)$ is infinite implies that the first case can not occur, and in the second case, $C=A=\alpha(C)$. In particular, $A$ is a finite group.

Lemma 4.2 Let $\pi: X \rightarrow Y$ be the orbit map of an injective $\mathbb{S}^{1}$-action. Then $\pi_{1}(X)$ is double-ended if and only if $\pi_{1}^{\mathrm{orb}}(Y)$ is finite.

Proof It suffices to show that if $\pi_{1}(X)$ is double-ended, then $\pi_{1}^{\text {orb }}(Y)$ is finite; the other direction is trivial; see eg Scott and Wall [42]. To see this, note that $\pi_{1}(X)=$ $A *_{A} \alpha$ for a finite group $A$ by Lemma 4.1, where we recall that $A *_{A} \alpha$ is generated by elements of $A$ and a letter $t$ with additional relations $t^{-1}=\alpha(a), a \in A$. If we let $H$ be the cyclic subgroup generated by $t$, then $H$ has finite index in $\pi_{1}(X)$. On the other hand, if we let $\Gamma$ be the subgroup generated by the homotopy class of a regular fiber of $\pi$, then $\Gamma \cap H$ has finite index in $H$ because $\alpha$ is of finite order. Consequently $\Gamma \cap H$ has finite index in $\pi_{1}(X)$. This implies that the index of $\Gamma$ in $\pi_{1}(X)$ is also finite, which means exactly that $\pi_{1}^{\text {orb }}(Y)$ is finite. Hence the lemma. 
Proof of Theorem 1.6 Part (1) The proof for this part is based on the rigidity of injective Seifert fibered space construction, which we shall briefly review first; see Lee and Raymond [28] for more details. Suppose we are given a group $\pi$ together with a short exact sequence $1 \rightarrow \Gamma \rightarrow \pi \rightarrow Q \rightarrow 1$, where $\Gamma=\mathbb{Z}^{k}$. Let $W$ be a simply connected smooth manifold and consider the trivial principal $\mathbb{R}^{k}$-bundle $\mathbb{R}^{k} \times W$ over $W$. Let $\psi$ be a smooth, free and properly discontinuous action of $\pi$ on $\mathbb{R}^{k} \times W$ via bundle morphisms, such that the restriction $\left.\psi\right|_{\Gamma}$ is given by translations via an embedding $\epsilon: \Gamma=\mathbb{Z}^{k} \rightarrow \mathbb{R}^{k}$ as a uniform lattice. Such an action $\psi$ induces a smooth action of $Q$ on $W$, which is denoted by $\rho$. The quotient space $E \equiv \mathbb{R}^{k} \times W / \psi(\pi)$ is a Seifert fibered space over the orbifold $W / \rho(Q)$, with regular fiber $T^{k}=\mathbb{R}^{k} / \epsilon(\Gamma)$ which is a $k$-dimensional torus. Conversely, a Seifert fibered space with a regular fiber $T^{k}$ must arise from such a construction if the inclusion of a regular fiber induces an injective map on $\pi_{1}$ (such Seifert fibered spaces are called injective). In this case the short exact sequence $1 \rightarrow \Gamma \rightarrow \pi \rightarrow Q \rightarrow 1$ is part of the homotopy exact sequence associated to the corresponding fibration, with $\pi$ being the $\pi_{1}$ of the Seifert fibered space, $\Gamma=\mathbb{Z}^{k}$ being the $\pi_{1}$ of a regular fiber, and $Q$ being the $\pi_{1}^{\text {orb }}$ of the base orbifold.

Given two such actions $\psi_{1}$ and $\psi_{2}$ of $\pi$, with induced embeddings $\epsilon_{1}, \epsilon_{2}: \Gamma \rightarrow \mathbb{R}^{k}$ and induced actions $\rho_{1}$ and $\rho_{2}$ of $Q$ on $W$, the aforementioned rigidity theorem asserts that if $\rho_{1}$ and $\rho_{2}$ are conjugate by a diffeomorphism $h: W \rightarrow W$, then $\psi_{1}$ and $\psi_{2}$ are conjugate by $(\lambda, g, h)$, where $\lambda \in C^{\infty}\left(W, \mathbb{R}^{k}\right), g \in \operatorname{GL}(k, \mathbb{R})$, and

$$
(\lambda, g, h) \cdot(v, w)=(g(v)+\lambda(h(w)), h(w)), \quad(v, w) \in \mathbb{R}^{k} \times W .
$$

Note that in particular, the corresponding Seifert fibered spaces $E_{1}=\mathbb{R}^{k} \times W / \psi_{1}(\pi)$ and $E_{2}=\mathbb{R}^{k} \times W / \psi_{2}(\pi)$ are diffeomorphic via a fiber-preserving diffeomorphism induced by $(\lambda, g, h)$; see [28, page 381$]$.

Now let $E_{1}$ and $E_{2}$ be two injective Seifert fibered spaces and let $\alpha: \pi_{1}\left(E_{1}\right) \rightarrow \pi_{1}\left(E_{2}\right)$ be an isomorphism. Furthermore, we assume that the universal covers of $E_{1}$ and $E_{2}$ are diffeomorphic, say given by $\mathbb{R}^{k} \times W$, and that the isomorphism $\alpha: \pi_{1}\left(E_{1}\right) \rightarrow \pi_{1}\left(E_{2}\right)$ respects the homotopy exact sequences associated to the corresponding fibrations on $E_{1}$ and $E_{2}$. Note that the latter is always true when there is a certain uniqueness of the short exact sequence $1 \rightarrow \Gamma \rightarrow \pi \rightarrow Q \rightarrow 1$, eg when $\Gamma=z(\pi)$. With this understood, we denote the group $\pi_{1}\left(E_{2}\right)$ by $\pi$ and identify $\pi_{1}(E)$ with $\pi$ via $\alpha$. Then $E_{1}$ and $E_{2}$ may be regarded as arising from the injective Seifert fibered space construction for some actions $\psi_{1}$ and $\psi_{2}$ of $\pi$ on $\mathbb{R}^{k} \times W$. Let $\rho_{1}$ and $\rho_{2}$ be the induced actions of $Q$ on $W$. Then the rigidity theorem mentioned above implies that there is a fiberpreserving diffeomorphism $\phi: E_{1} \rightarrow E_{2}$ such that $\phi_{*}=\alpha: \pi_{1}\left(E_{1}\right) \rightarrow \pi_{1}\left(E_{2}\right)$ if $\rho_{1}$ and $\rho_{2}$ are conjugate by a diffeomorphism $h: W \rightarrow W$. (Roughly speaking, the above 
rigidity theorem allows us to show that if the diffeomorphism classification of the base orbifolds are determined by the fundamental groups, then so are the fiber-preserving diffeomorphism classification of the corresponding Seifert fibered spaces.)

With the preceding understood, we shall now give a proof for part (1). Consider first the case where rank $z\left(\pi_{1}(X)\right)>1$. A smooth classification of such fixed-point free, smooth $\mathbb{S}^{1}$-four-manifolds is given in Theorem 4.3, which shows that it suffices to consider the case where rank $z\left(\pi_{1}(X)\right)=2$ and $\pi_{2}(X)=0$. Moreover, it also shows that, in this case, $X$ and $X^{\prime}$ arise from the above injective Seifert fibered space construction with $k=2$ and $W=\mathbb{R}^{2}$. (Note that the uniqueness of the short exact sequence follows from the fact that $\Gamma=z(\pi)$; see Lemma 2.2(a).) With this understood, the existence of $\phi: X \rightarrow X^{\prime}$ with $\phi_{*}=\alpha$ follows from the fact that for orientable 2 -orbifolds with infinite fundamental group, any isomorphism of $\pi_{1}^{\text {orb }}$ may be realized by a diffeomorphism of the 2-orbifolds; eg see [29].

It remains to consider the case where rank $z\left(\pi_{1}(X)\right)=1$. In this case, $X$ is an injective Seifert fibered space over a 3 -orbifold $Y$ with regular fiber $\mathbb{S}^{1}$, where $Y$ is an irreducible 3-orbifold with infinite fundamental group. As $Y$ is good, the Geometrization theorem implies that $Y=\tilde{Y} / G$ for some aspherical 3-manifold $\tilde{Y}$; see $[31 ; 4]$. (Note that $G$ may be trivial here.) Furthermore, by the Geometrization theorem, $\widetilde{Y}$ admits a geometric decomposition; see eg Kleiner and Lott [27]. In particular, $\tilde{Y}$ is either Haken, or Seifert fibered, or hyperbolic, and the universal cover of $\tilde{Y}$ is diffeomorphic to $\mathbb{R}^{3}$. With this understood, we see that $X$ arises from the injective Seifert fibered space construction with $k=1$ and $W=\mathbb{R}^{3}$. (Note that the condition $\Gamma=z(\pi)$ is satisfied (see Lemma 2.3), which gives the required uniqueness for the short exact sequence $1 \rightarrow \Gamma \rightarrow \pi \rightarrow Q \rightarrow 1$.) It remains to show that for irreducible 3 -orbifolds with infinite fundamental group, any isomorphism of $\pi_{1}^{\text {orb }}$ may be realized by a diffeomorphism of the 3 -orbifolds. This was verified by McCullough and Miller (see the proof of Corollary 5.3 in [31]) when $\tilde{Y}$ is either Haken or Seifert fibered. For the remaining case, the 3-orbifolds are hyperbolic, and in this case, Mostow Rigidity implies that any isomorphism of $\pi_{1}^{\text {orb }}$ may be realized by an isometry of the 3-orbifolds. This finishes off the proof for part (1).

Part (2): Let $\pi: X \rightarrow Y$ be the orbit map of the $\mathbb{S}^{1}$-action on $X$. By Lemma 4.2, this is the case precisely when $Y$ has finite fundamental group. By the Geometrization theorem, $Y$ is a spherical 3-orbifold, ie there is a finite subgroup $G$ of $\operatorname{SO}(4)$ such that $Y=\mathbb{S}^{3} / G$. Note that the Euler class of $\pi: X \rightarrow Y$ is torsion, so that there is a 3-manifold $\hat{Y}$ and a periodic diffeomorphism $f$ such that $Y=\hat{Y} /\langle f\rangle$ and $X$ is the mapping torus of $f$. Moreover, by the Geometrization theorem, $\hat{Y}$ is an elliptic 3 -manifold. Similar conclusions hold for $X^{\prime}$; ie $X^{\prime}$ is the mapping torus of a periodic diffeomorphism $f^{\prime}$ of an elliptic 3-manifold $\hat{Y}^{\prime}$. 
Note that the mapping torus descriptions of $X$ and $X^{\prime}$ imply that $\pi_{1}(X)$ and $\pi_{1}\left(X^{\prime}\right)$ are given by HNN extensions $\pi_{1}(\hat{Y}) *_{\pi_{1}(\hat{Y})} f_{*}$ and $\pi_{1}\left(\hat{Y}^{\prime}\right) *_{\pi_{1}\left(\hat{Y}^{\prime}\right)} f_{*}^{\prime}$, respectively. This gives rise to short exact sequences

$$
1 \rightarrow \pi_{1}(\hat{Y}) \stackrel{i}{\rightarrow} \pi_{1}(X) \stackrel{p}{\rightarrow} \mathbb{Z} \rightarrow 1 \quad \text { and } \quad 1 \rightarrow \pi_{1}\left(\hat{Y}^{\prime}\right) \stackrel{i^{\prime}}{\rightarrow} \pi_{1}\left(X^{\prime}\right) \stackrel{p^{\prime}}{\rightarrow} \mathbb{Z} \rightarrow 1 .
$$

With this understood, given any isomorphism $\alpha: \pi_{1}(X) \rightarrow \pi_{1}\left(X^{\prime}\right)$, we observe that the homomorphism $p^{\prime} \circ \alpha \circ i: \pi_{1}(\hat{Y}) \rightarrow \mathbb{Z}$ is trivial because $\pi_{1}(\hat{Y})$ is finite. This implies that $\alpha \circ i: \pi_{1}(\hat{Y}) \rightarrow \pi_{1}\left(X^{\prime}\right)$ lies in the image of $i^{\prime}: \pi_{1}\left(\hat{Y}^{\prime}\right) \rightarrow \pi_{1}\left(X^{\prime}\right)$. It follows easily from this consideration that $\alpha: \pi_{1}(X) \rightarrow \pi_{1}\left(X^{\prime}\right)$ induces an isomorphism $\hat{\alpha}: \pi_{1}(\hat{Y}) \rightarrow \pi_{1}\left(\hat{Y}^{\prime}\right)$ such that $f_{*}^{\prime}=\hat{\alpha} \circ f_{*} \circ \hat{\alpha}^{-1}$ as an element of Out $\left(\pi_{1}\left(\hat{Y}^{\prime}\right)\right)$. Suppose $\hat{\alpha}$ can be realized by a diffeomorphism $h: \hat{Y} \rightarrow \hat{Y}^{\prime}$, eg when $\hat{Y}$ and $\hat{Y}^{\prime}$ are not lens spaces. Identifying $\hat{Y}$ with $\hat{Y}^{\prime}$ via $h, X$ may be regarded as the mapping torus of the periodic diffeomorphism $g=h \circ f \circ h^{-1}: \hat{Y}^{\prime} \rightarrow \hat{Y}^{\prime}$. Now observe that $g_{*}=f_{*}^{\prime}$ as an element of Out $\left(\pi_{1}\left(\hat{Y}^{\prime}\right)\right)$, which implies that $g$ and $f^{\prime}$ are homotopic, hence isotopic; see $[1 ; 39 ; 7 ; 26 ; 40 ; 6]$. The existence of $\phi: X \rightarrow X^{\prime}$ with $\phi_{*}=\alpha$ follows easily from these considerations. This finishes the proof of part (2).

We end this section with the smooth classification theorem alluded to earlier. The proof of the theorem employs a key lemma, Lemma 5.2, whose proof will be given in the next section.

Theorem 4.3 Suppose that $X$ is a fixed-point free, smooth $\mathbb{S}^{1}$-four-manifold with rank $z\left(\pi_{1}(X)\right)>1$. Then $X$ belongs to one of the following cases:

(1) If rank $z\left(\pi_{1}(X)\right)>2$, then $X$ is diffeomorphic to the 4-torus $T^{4}$.

(2) If rank $z\left(\pi_{1}(X)\right)=2$ and $\pi_{2}(X) \neq 0$, then $X$ is diffeomorphic to $T^{2} \times \mathbb{S}^{2}$.

(3) If rank $z\left(\pi_{1}(X)\right)=2$ and $\pi_{2}(X)=0$, then $X$ is diffeomorphic to $\mathbb{S}^{1} \times N^{3} / G$, where $N^{3}$ is an irreducible Seifert 3-manifold with infinite fundamental group, and $G$ is a finite cyclic group acting on $\mathbb{S}^{1} \times N^{3}$ preserving the product structure and orientation on each factor, and the Seifert fibration on $N^{3}$.

Proof Let $\pi: X \rightarrow Y$ be the orbit map of the $\mathbb{S}^{1}$-action. Note that $\pi_{*}: \pi_{1}(X) \rightarrow$ $\pi_{1}^{\text {orb }}(Y)$ is surjective, so that $\pi_{*}\left(z\left(\pi_{1}(X)\right)\right.$ is contained in $z\left(\pi^{\mathrm{orb}}(Y)\right)$. It follows easily from rank $z\left(\pi_{1}(X)\right)>1$ that $z\left(\pi^{\text {orb }}(Y)\right)$ is infinite. By Lemma 5.2, $Y$ is Seifert fibered, and furthermore, by Lemma 2.6, $\pi: X \rightarrow Y$ extends to a principal $T^{2}$-bundle over a 2 -orbifold $B$, which will be denoted by $\Pi: X \rightarrow B$. We remark that $B$ is an orientable, closed 2 -orbifold.

We begin by describing a decomposition of the principal $T^{2}$-bundle into a pair of principal $\mathbb{S}^{1}$-bundles over $B$. More concretely, given any basis $\left(e_{1}, e_{2}\right)$ of $\pi_{1}\left(T^{2}\right)$, 
we let $\theta_{i}: T^{2} \rightarrow \mathbb{S}^{1}, i=1,2$, be the projections to the first and the second factor of the decomposition $T^{2}=\mathbb{S}^{1} \times \mathbb{S}^{1}$ that is determined by the basis $\left(e_{1}, e_{2}\right)$. This gives rise to a pair of principal $\mathbb{S}^{1}$-bundles over $B$, denoted by $V_{1}$ and $V_{2}$, which are induced by $\theta_{1}$ and $\theta_{2}$, respectively. Note that one can recover the principal $T^{2}$-bundle $\Pi: X \rightarrow B$ as the pull-back bundle of $V_{1} \times V_{2} \rightarrow B \times B$ via the diagonal map $B \rightarrow B \times B$. Moreover, with a change of basis, one can always arrange $V_{1}$ to have vanishing Euler number. Indeed, under the change of basis

$$
e_{1}=a e_{1}^{\prime}+c e_{2}^{\prime}, \quad e_{2}=b e_{1}^{\prime}+d e_{2}^{\prime},
$$

where $a d-b c=1$, the corresponding principal $\mathbb{S}^{1}$-bundles $V_{1}^{\prime}$ and $V_{2}^{\prime}$ associated to the basis $\left(e_{1}^{\prime}, e_{2}^{\prime}\right)$ have Euler numbers

$$
e\left(V_{1}^{\prime}\right)=a \cdot e\left(V_{1}\right)+b \cdot e\left(V_{2}\right), \quad e\left(V_{2}^{\prime}\right)=c \cdot e\left(V_{1}\right)+d \cdot e\left(V_{2}\right) .
$$

If both of $e\left(V_{1}\right)$ and $e\left(V_{2}\right)$ are nonzero, one can choose a unique pair of integers (up to a sign), $(a, b)$, such that $e\left(V_{1}^{\prime}\right)=0$. Note that, up to a sign, $e\left(V_{2}^{\prime}\right)$ is independent of the choices of $c$ and $d$. This said, we shall assume in what follows that $e\left(V_{1}\right)=0$.

With these preparations, we now consider case (1) where rank $z\left(\pi_{1}(X)\right)>2$. It is clear that $z\left(\pi_{1}^{\text {orb }}(B)\right)$ is nontrivial and infinite. By Lemma 2.2(a), B must be a nonsingular torus. As $e\left(V_{1}\right)=0$ and $B$ is nonsingular, $V_{1}$ is trivial, which implies that $X=\mathbb{S}^{1} \times V_{2}$. Finally, the assumption that rank $z\left(\pi_{1}(X)\right)>2$ implies that $V_{2}$ must also be trivial. Hence $X$ is diffeomorphic to the 4-torus $T^{4}$.

Consider case (2) where rank $z\left(\pi_{1}(X)\right)=2$ and $\pi_{2}(X) \neq 0$. Note that $X$ is a principal $\mathbb{S}^{1}$-bundle over $V_{2}$, which is the pull-back of the principal $\mathbb{S}^{1}$-bundle $V_{1} \rightarrow B$ via the map $V_{2} \rightarrow B$. The homotopy exact sequence associated to the fibration $X \rightarrow V_{2}$ (see Haefliger [22]) implies that $z\left(\pi_{1}^{\text {orb }}\left(V_{2}\right)\right)$ is infinite and $\pi_{2}^{\text {orb }}\left(V_{2}\right) \neq 0$. By Lemma 5.2, $V_{2}$ is the mapping torus of a periodic diffeomorphism of a 2 -orbifold $\Sigma$ where $\pi_{1}^{\text {orb }}(\Sigma)$ is finite. Now observe that $e\left(V_{1}\right)=0$ implies that $\Sigma$ must be either $\mathbb{S}^{2}$ or a football. It follows easily that $X$ is diffeomorphic to $T^{2} \times \mathbb{S}^{2}$, which finishes the proof for case (2).

For case (3) where rank $z\left(\pi_{1}(X)\right)=2$ and $\pi_{2}(X)=0$, we first observe that $\pi_{1}^{\text {orb }}(B)$ is infinite, and therefore $B$ is good. Let $B=\widetilde{B} / \Gamma$, where $\widetilde{B}$ is a closed orientable surface and $\Gamma$ is a finite group acting on $\widetilde{B}$. We let $\widetilde{X}, \widetilde{V}_{1}$, and $\widetilde{V}_{2}$ be the pull-backs of $X \rightarrow B, V_{1} \rightarrow B$, and $V_{2} \rightarrow B$ to $\widetilde{B}$ via $\widetilde{B} \rightarrow B=\widetilde{B} / \Gamma$. Then $\Gamma$ acts freely on $\tilde{X}$, giving $X=\tilde{X} / \Gamma$ and $\widetilde{V}_{1}=\mathbb{S}^{1} \times \widetilde{B}$. Let $\Gamma_{1}$ be the subgroup of $\Gamma$ which acts trivially on the $\mathbb{S}^{1}$-factor in $\widetilde{V}_{1}=\mathbb{S}^{1} \times \widetilde{B}$. Then $\Gamma_{1}$ acts freely on $\widetilde{V}_{2}$. Denote by $N^{3}$ the quotient $\widetilde{V}_{2} / \Gamma_{1}$, which is clearly an irreducible Seifert 3 -manifold with infinite fundamental group. With this understood, note that $\tilde{X} / \Gamma_{1}=\mathbb{S}^{1} \times N^{3}$, so that if we 
set $G=\Gamma / \Gamma_{1}$, then $X=\mathbb{S}^{1} \times N^{3} / G$ where the action of $G$ preserves the product structure and the orientation of each factor, as well as the Seifert fibration on $N^{3}$, as claimed. This finishes the proof of Theorem 4.3.

Remark 4.4 Theorem 2.1 in [12] asserts that if a 4-manifold with $b_{2}^{+} \geq 1$ has nontrivial Seiberg-Witten invariant, then the homotopy class of the principal orbits of any smooth, fixed-point free $\mathbb{S}^{1}$-action on the manifold must be of infinite order; in particular, the center of the fundamental group must be infinite. As a corollary of Theorem 4.3(2), the converse of the above statement is not true. More concretely, consider a ruled surface $X$ which is a nontrivial $\mathbb{S}^{2}$-bundle over $T^{2}$. Note that $X$ satisfies $b_{2}^{+} \geq 1$, it has nontrivial Seiberg-Witten invariant, and $z\left(\pi_{1}(X)\right)$ is infinite. However, by Theorem 4.3(2), $X$ does not admit any smooth, fixed-point free $\mathbb{S}^{1}$ action. It is also interesting to note that a double cover of $X$, which is diffeomorphic to $\mathbb{S}^{2} \times T^{2}$, admits a smooth, fixed-point free $\mathbb{S}^{1}$-action. We remark that for a closed aspherical manifold, such a correlation between the existence of circle actions and the nontriviality of the center of the fundamental group is part of a conjectured rigidity of aspherical manifolds going back to work of Borel. See [8] for some recent progress and more detailed discussions.

\section{Injectivity of $\mathbb{S}^{1}$-actions when $\pi_{1}$ has infinite center}

The main purpose of this section is to show that a smooth fixed-point free $\mathbb{S}^{1}$-fourmanifold whose fundamental group has infinite center is injective, and hence admits a fiber-sum decomposition. A key role is played by Lemma 5.2, whose proof requires the use of the Geometrization theorem in various forms.

We begin with the following observation.

Lemma 5.1 Let $Y$ be a 3-orbifold with a singular set consisting of a union of circles. Then there is a good 3-orbifold $Y_{0}$ such that $Y$ and $Y_{0}$ have the same underlying space, and $\pi_{1}^{\text {orb }}\left(Y_{0}\right)=\pi_{1}^{\text {orb }}(Y)$.

Proof Denote by $|Y|$ the underlying 3-manifold of $Y$ and by $\Sigma Y$ the singular set of $Y$, consisting of components $\gamma_{1}, \ldots, \gamma_{n}$. Then $\pi_{1}^{\text {orb }}(Y)$ admits the following presentation

$$
\pi_{1}^{\mathrm{orb}}(Y)=\pi_{1}(|Y| \backslash \Sigma Y) / N .
$$

Here $N$ is the normal subgroup generated by the elements $\mu_{\gamma_{i}}^{m_{i}}, i=1,2, \ldots, n$, where $\mu_{\gamma_{i}}$ is the meridian around $\gamma_{i}$ and $m_{i}$ is the multiplicity of $\gamma_{i}$; see [5, Proposition 2.7].

With this understood, for any bad 2-suborbifold $C$ in $Y$, one has the following two possibilities: 
(i) There is exactly one $\gamma_{i}$ such that $C \cap \gamma_{i} \neq \varnothing$.

(ii) There are $\gamma_{i}$ and $\gamma_{j}$, with $i \neq j$ and $m_{i} \neq m_{j}$, such that $C \cap \gamma_{i} \neq \varnothing$ and $C \cap \gamma_{j} \neq \varnothing$.

In case (i), the existence of such a $C$ implies that $\mu_{\gamma_{i}}=1$ in $\pi_{1}(|Y| \backslash \Sigma Y)$, hence $\pi_{1}^{\text {orb }}(Y)$ is unchanged after removing $\gamma_{i}$ from $\Sigma Y$. In the resulting 3 -orbifold, $C$ is no longer a bad 2-suborbifold.

In case (ii), let $m=\operatorname{gcd}\left(m_{i}, m_{j}\right)$. We change $Y$ to a new 3 -orbifold by replacing the multiplicities of $\gamma_{i}, \gamma_{j}$ with $m$. (In case of $m=1$, this simply means that $\gamma_{i}, \gamma_{j}$ are both removed from $\Sigma Y$.) Note that the existence of $C$ implies that the normal subgroup generated by $\mu_{\gamma_{i}}^{m_{i}}$ and $\mu_{\gamma_{j}}^{m_{j}}$ is the same as that generated by $\mu_{\gamma_{i}}^{m}$ and $\mu_{\gamma_{j}}^{m}$. It follows that $\pi_{1}^{\text {orb }}(Y)$ remains unchanged in this process. Since there are only finitely many singular circles and during the process either the number of singular circles is decreased or the multiplicity of a singular circle is decreased, this process must terminate in finitely many steps. At the end, we obtain a good 3-orbifold $Y_{0}$ such that $\left|Y_{0}\right|=|Y|$ and $\pi_{1}^{\text {orb }}\left(Y_{0}\right)=\pi_{1}^{\text {orb }}(Y)$. Hence the lemma.

A more conceptual view which was suggested by the referee goes as follows: introducing a notion of complexity for 3-orbifolds, say by the sum of the multiplicities of the singular circles, then the orbifold $Y_{0}$ in Lemma 5.1 is characterized as the one with the minimal complexity among the 3-orbifolds which have the same underlying space and the same fundamental group of the orbifold $Y$.

In the following lemma, for the definition of $\pi_{2}^{\text {orb }}(Y)$ we refer to $[21 ; 22 ; 10]$.

Lemma 5.2 Let $Y$ be an orientable 3-orbifold, not necessarily good, with a singular set consisting of a union of circles. If $z\left(\pi_{1}^{\mathrm{orb}}(Y)\right)$ is infinite, then $Y$ is Seifert fibered. Moreover, if $\pi_{2}^{\text {orb }}(Y) \neq 0$, then $Y$ is the mapping torus of a periodic diffeomorphism of a 2 -orbifold with finite fundamental group.

Proof Let $Y_{0}$ be the good 3-orbifold associated to $Y$ from Lemma 5.1, which is clearly orientable. Then there is an orientable 3 -manifold $Y^{\prime}$ equipped with a finite group action of $G$, such that $Y_{0}=Y^{\prime} / G$; see $[4 ; 31]$. Since $\pi_{1}^{\text {orb }}\left(Y_{0}\right)=$ $\pi_{1}^{\text {orb }}(Y), z\left(\pi_{1}^{\text {orb }}\left(Y_{0}\right)\right)$ is also infinite, and consequently, $z\left(\pi_{1}\left(Y^{\prime}\right)\right)$, which contains $\pi_{1}\left(Y^{\prime}\right) \cap z\left(\pi_{1}^{\text {orb }}\left(Y_{0}\right)\right)$, is infinite. As an abelian subgroup of a 3-manifold group, $z\left(\pi_{1}\left(Y^{\prime}\right)\right)$ must contain an infinite cyclic subgroup $H$ (see [24, Theorem 9.14]), which is clearly normal in $\pi_{1}\left(Y^{\prime}\right)$.

Consider first the case where $\pi_{2}\left(Y^{\prime}\right)=0$. By work of Gabai (see [20] and, independently, Casson and Jungreis [9]), $Y^{\prime}$ is Seifert fibered, with $H$ being generated by 
a regular fiber of the Seifert fibration. Since $H \subset z\left(\pi_{1}^{\text {orb }}\left(Y_{0}\right)\right)$, it must be invariant under the action of $G$. By a theorem of Meeks and Scott (see [32, Theorem 2.2]), $G$ preserves the Seifert fibration on $Y^{\prime}$, which implies that $Y_{0}$ is Seifert fibered. Since we assume $\pi_{2}\left(Y^{\prime}\right)=0, Y_{0}$ does not contain any essential spherical 2-suborbifold. From the proof of Lemma 5.1, we see that $Y$ contains no bad 2-suborbifold, and in this case, $Y=Y_{0}$. This proves that $Y$ is Seifert fibered. Note that in this case,

$$
\pi_{2}^{\mathrm{orb}}(Y)=\pi_{2}^{\mathrm{orb}}\left(Y_{0}\right)=\pi_{2}\left(Y^{\prime}\right)=0 .
$$

Suppose $\pi_{2}\left(Y^{\prime}\right) \neq 0$. Since $z\left(\pi_{1}\left(Y^{\prime}\right)\right)$ is nontrivial, $Y^{\prime}$ must be prime (here we use Lemma 2.1 and the resolution of the Poincaré conjecture [36]), and consequently, $Y^{\prime}=\mathbb{S}^{1} \times \mathbb{S}^{2}$. Note that $G$ must act on $Y^{\prime}=\mathbb{S}^{1} \times \mathbb{S}^{2}$ homologically trivially because the fundamental group of $Y_{0}=Y^{\prime} / G$ is infinite. By Lemma 2.5, $Y_{0}=Y^{\prime} / G$ is the mapping torus of a periodic diffeomorphism of some spherical 2-orbifold; in particular, $Y_{0}$ is Seifert fibered. If $Y$ is good, then $Y=Y_{0}$, and the lemma follows in this case. Note that in this case,

$$
\pi_{2}^{\mathrm{orb}}(Y)=\pi_{2}^{\mathrm{orb}}\left(Y_{0}\right)=\pi_{2}\left(Y^{\prime}\right) \neq 0 .
$$

It remains to consider the case where $Y$ is not good. Recall that in the proof of Lemma 5.1, $Y_{0}$ is obtained from $Y$ by performing a sequence of operations where, in each, either a singular circle is removed or its multiplicity is decreased. Since $Y_{0}$ is the mapping torus of a periodic diffeomorphism $f$ of some spherical 2-orbifold $\Sigma$, it follows easily that $\Sigma$ is either $\mathbb{S}^{2}$ or a football. Moreover, if $\Sigma$ is a football, $f$ must be isotopic to the identity map, and therefore $Y_{0}$ is diffeomorphic to $\mathbb{S}^{1} \times \Sigma$. It follows readily that $Y$ is the product of $\mathbb{S}^{1}$ with a bad 2-orbifold $B$. Note that in this case,

$$
\pi_{2}^{\mathrm{orb}}(Y)=\pi_{2}^{\mathrm{orb}}(B) \neq 0,
$$

since a bad 2-orbifold has nontrivial $\pi_{2}^{\text {orb }}$.

Suppose $\Sigma=\mathbb{S}^{2}$, and therefore $Y_{0}=\mathbb{S}^{1} \times \mathbb{S}^{2}$. Note that $Y$ can have at most two singular circles. Assume first that $Y$ has only one singular circle, which is denoted by $\gamma$. It suffices to show that $(|Y|, \gamma)$ and $\left(\mathbb{S}^{1} \times \mathbb{S}^{2}, \mathbb{S}^{1} \times\{\mathrm{pt}\}\right)$ are diffeomorphic. To see this, let $W=Y \backslash N d(\gamma)$ and let $\mu$ denote a meridian of $\gamma$. Then $\pi_{1}^{\text {orb }}(Y)=$ $\pi_{1}(W) /\left\langle\mu^{m}\right\rangle$ where $m$ denotes the multiplicity of $\gamma$. Since $\mu$ bounds a disc in $W$, and $\pi_{1}^{\text {orb }}(Y)=\pi_{1}\left(Y_{0}\right)=\mathbb{Z}$, it follows that $\pi_{1}(W)=\mathbb{Z}$. Cutting $W$ open along the disc bounded by $\mu$, we obtain a $3-$ manifold $W_{0}$ with $\partial W_{0}=\mathbb{S}^{2}$ and $\pi_{1}\left(W_{0}\right)$ trivial. By the Geometrization theorem, $W_{0}$ is a 3-ball, which implies easily that $(|Y|, \gamma)$ is diffeomorphic to $\left(\mathbb{S}^{1} \times \mathbb{S}^{2}, \mathbb{S}^{1} \times\{\mathrm{pt}\}\right)$. This shows that $Y$ is the product of $\mathbb{S}^{1}$ with a teardrop. Note that $\pi_{2}^{\text {orb }}(Y) \neq 0$ as we argued before. 
Finally, suppose $Y$ has two components, denoted by $\gamma_{1}, \gamma_{2}$, which have multiplicities $m_{1}, m_{2}$ respectively. From the construction of $Y_{0}$ in Lemma 5.1, it follows easily that $m_{1}, m_{2}$ are relatively prime. With this understood, it suffices to show that $\left(|Y|, \gamma_{1}, \gamma_{2}\right)$ is diffeomorphic to $\left(\mathbb{S}^{1} \times \mathbb{S}^{2}, \mathbb{S}^{1} \times\{\mathrm{pt}\}, \mathbb{S}^{1} \times\{\mathrm{pt}\}\right)$. First, as we argued in the previous case, $\left(|Y|, \gamma_{1}\right)$ is diffeomorphic to $\left(\mathbb{S}^{1} \times \mathbb{S}^{2}, \mathbb{S}^{1} \times\{\mathrm{pt}\}\right)$, so that if we let $W=Y \backslash$ $N d\left(\gamma_{1}\right)$, then $|W|=\mathbb{S}^{1} \times D^{2}$. It remains to show that $\left(|W|, \gamma_{2}\right)$ is diffeomorphic to $\left(\mathbb{S}^{1} \times D^{2}, \mathbb{S}^{1} \times\{\mathrm{pt}\}\right)$. To see this, note that the meridians $\mu_{1}$ and $\mu_{2}$ of $\gamma_{1}$ and $\gamma_{2}$, respectively, bound an annulus in $W \backslash N d\left(\gamma_{2}\right)$. Consequently,

$$
\mathbb{Z}=\pi_{1}^{\text {orb }}(Y)=\pi_{1}\left(W \backslash N d\left(\gamma_{2}\right)\right) /\left\langle\mu_{1}^{m_{1}}, \mu_{2}^{m_{2}}\right\rangle=\pi_{1}\left(W \backslash N d\left(\gamma_{2}\right)\right) /\left\langle\mu_{2}\right\rangle,
$$

which implies that the following sequence is short exact:

$$
1 \rightarrow \mathbb{Z}_{m_{2}} \rightarrow \pi_{1}^{\text {orb }}(W)=\pi_{1}\left(W \backslash N d\left(\gamma_{2}\right)\right) /\left\langle\mu_{2}^{m_{2}}\right\rangle \rightarrow \mathbb{Z} \rightarrow 1 .
$$

Now if we cut $W$ open along a copy of $\{\mathrm{pt}\} \times D^{2}$ in $|W|=\mathbb{S}^{1} \times D^{2}$, we obtain a 3-orbifold $W_{0}$ with $\partial W_{0}=\mathbb{S}^{2} / \mathbb{Z}_{m_{2}}$. Moreover, it follows from the above short exact sequence that $\pi_{1}^{\text {orb }}\left(W_{0}\right)=\mathbb{Z}_{m_{2}}$. Then the Geometrization theorem implies that $W_{0}$ is discal, from which it follows that $\left(|Y|, \gamma_{1}, \gamma_{2}\right)$ is diffeomorphic to $\left(\mathbb{S}^{1} \times \mathbb{S}^{2}, \mathbb{S}^{1} \times\right.$ $\left.\{\mathrm{pt}\}, \mathbb{S}^{1} \times\{\mathrm{pt}\}\right)$, and consequently, $Y$ is the product of $\mathbb{S}^{1}$ with a bad 2-orbifold. Moreover, $\pi_{2}^{\text {orb }}(Y) \neq 0$. This finishes the proof of the lemma.

Proof of Theorem 1.4 Let $\pi: X \rightarrow Y$ be the orbit map of the fixed-point free $\mathbb{S}^{1}$-action. Suppose the $\mathbb{S}^{1}$-action is not injective. Then the homotopy class of a regular fiber of $\pi$ is finite, and since $z\left(\pi_{1}(X)\right)$ is infinite, the image of $z\left(\pi_{1}(X)\right)$ under $\pi_{*}: \pi_{1}(X) \rightarrow \pi_{1}^{\text {orb }}(Y)$, clearly contained in $z\left(\pi_{1}^{\text {orb }}(Y)\right)$, must also be infinite. By Lemma 5.2, either $Y$ is irreducible, or $Y$ is the mapping torus of a periodic diffeomorphism of a 2-orbifold with finite fundamental group. Since we assume that the homotopy class of a regular fiber of $\pi$ is finite, $Y$ can not be irreducible. Then it follows easily that $X$ is the mapping torus of a periodic diffeomorphism of some elliptic 3-manifold.

To see that $X$ admits a fiber-sum decomposition, it suffices to consider the case where the $\mathbb{S}^{1}$-action is injective. We note first that the fact that the homotopy class of a regular fiber of $\pi$ has infinite order implies that the orbit space $Y$ of the $\mathbb{S}^{1}$-action does not contain any bad 2-suborbifolds. In other words, $Y$ must be good. By Lemma 2.4, $Y$ admits a reduced spherical decomposition. More precisely, there is a system of finitely many spherical 2-suborbifolds $\Sigma_{j} \subset Y$ such that, after capping off the boundary of each component of $Y \backslash \bigcup_{j} \Sigma_{j}$, one obtains a collection of 3-orbifolds $Y_{i}$ where each $Y_{i}$ is irreducible. Furthermore, each $\Sigma_{j}$ must be either an ordinary 2-sphere or a football, and the preimage $N_{j} \equiv \pi^{-1}\left(\Sigma_{j}\right)$ must be diffeomorphic to $\mathbb{S}^{1} \times \mathbb{S}^{2}$, because 
the homotopy class of a regular fiber of $\pi$ has infinite order. Finally, observe that the restriction of $\pi$ on each $N_{j}$ may be uniquely extended to a Seifert-type $\mathbb{S}^{1}$-fibration on $\mathbb{S}^{1} \times B^{3}$ so that, correspondingly, we obtain the irreducible $\mathbb{S}^{1}$-four-manifolds $X_{i}$ and the orbit maps $\pi_{i}: X_{i} \rightarrow Y_{i}$. It follows easily that $X$ is fiber-sum-decomposed into $X_{i}$ along $N_{j}$. We remark that the requirement that the spherical decomposition of $Y$ be reduced ensures that Definition 1.3(iv) is satisfied. This finishes off the proof of Theorem 1.4.

Proof of Corollary 1.7 By Theorem 1.4, it suffices to consider the case where the $\mathbb{S}^{1}$-action is injective. Let $\pi: X \rightarrow Y$ be the corresponding orbit map. We observe that $Y$ does not contain any bad 2-suborbifolds, hence there exist a 3-manifold $\tilde{Y}$ and a finite group $G$ such that $Y=\tilde{Y} / G$; see $[4 ; 31]$. On the other hand, by the homotopy exact sequence associated to $\pi: X \rightarrow Y$ (see Haefliger [22]), it follows easily that $\pi_{*}: \pi_{2}(X) \rightarrow \pi_{2}^{\text {orb }}(Y)$ is an isomorphism. Let $\tilde{\pi}: \tilde{X} \rightarrow \tilde{Y}$ be the pull-back fibration via the projection $\tilde{Y} \rightarrow Y$. Then $\tilde{X}$ is a finite regular cover of $X$. It suffices to show that there exist no embedded 2 -spheres with odd self-intersection in $\tilde{X}$.

Suppose to the contrary, there is an embedded 2 -sphere $C$ in $\tilde{X}$ with $C^{2} \equiv 1(\bmod 2)$. Consider the projection of $C$ into $\tilde{Y}$ under $\tilde{\pi}$. Clearly $[C] \in \pi_{2}(\tilde{X})$ is nonzero. On the other hand, $\pi_{*}: \pi_{2}(X) \rightarrow \pi_{2}^{\text {orb }}(Y)$ is an isomorphism, so that $\tilde{\pi}_{*}: \pi_{2}(\tilde{X}) \rightarrow \pi_{2}(\tilde{Y})$ is also an isomorphism. Consequently, $\left.\tilde{\pi}\right|_{C}: \mathbb{S}^{2} \rightarrow \tilde{Y}$ is homotopically nontrivial. By the Sphere theorem (see [24, Theorem 4.11]), there is an embedded 2-sphere $\Sigma$ in a neighborhood of $\tilde{\pi}(C)$, whose class is clearly homologous to $\tilde{\pi}_{*}[C]$. Observe that the Euler class of $\tilde{\pi}: \tilde{X} \rightarrow \tilde{Y}$ evaluates to 0 on $\Sigma$. This is because the pull-back of the Euler class of $\tilde{\pi}$ to $\tilde{X}$ is zero so that the Euler class of $\tilde{\pi}$ evaluates trivially on the class of $\tilde{\pi}(C)$. This implies that the restriction of $\tilde{\pi}$ to $\Sigma$ is trivial, and in particular, $\Sigma$ has a section $\Sigma^{\prime}$ in $\tilde{X}$. Consequently, we obtain an equation of homology classes

$$
C=\Sigma^{\prime}+\sum_{i} T_{i}
$$

where $T_{i}=\tilde{\pi}^{-1}\left(\gamma_{i}\right)$ for some loops $\gamma_{i} \subset \tilde{Y}$; see [2, Theorem 9]. Since $\Sigma^{\prime}$ and all $T_{i}$ have self-intersection 0 , this implies $C^{2} \equiv 0(\bmod 2)$, which is a contradiction. This finishes the proof of Corollary 1.7 .

\section{Theorems 1.1 and 1.2}

This section is devoted to the proofs of Theorems 1.1 and 1.2. We remark that while Theorem 1.1 follows readily from Theorems 1.5 and 1.6, the proof of Theorem 1.2 requires some additional care in the case when each irreducible $\mathbb{S}^{1}$-four-manifold in 
the fiber-sum decomposition is a mapping torus of a periodic diffeomorphism of a lens space. Furthermore, the case when the fundamental group of the 4-manifold is isomorphic to the fundamental group of a Klein bottle needs to be dealt with separately. In all these considerations, the following lemma describing certain isotopies of periodic diffeomorphisms of $\mathbb{S}^{3}$ or a lens space plays a key role.

Let $Y=\mathbb{S}^{3} / G$ where $G$ is a cyclic subgroup of $\mathrm{SO}(4)$ of order $n$ given by

$$
\lambda \cdot\left(z_{1}, z_{2}\right)=\left(\lambda^{p} z_{1}, \lambda^{q} z_{2}\right),
$$

where $\lambda=\exp (2 \pi i / n)$ is a $n^{\text {th }}$ root of unity and $\operatorname{gcd}(n, p, q)=1$. Set $u=\operatorname{gcd}(n, p)$, $v=\operatorname{gcd}(n, q)$. Then $\operatorname{gcd}(u, v)=1$ so that $u v$ is a divisor of $n=\left|\pi_{1}^{\text {orb }}(Y)\right|$, and $Y$ has at most two singular circles of multiplicities $u$ and $v$, given by $z_{2}=0$ and $z_{1}=0$, respectively.

Suppose $H$ is a subgroup of $G$ of order $\hat{n}$ generated by $\lambda^{n / \hat{n}}$, which acts freely on $\mathbb{S}^{3}$. Note that this condition is equivalent to $\operatorname{gcd}(\hat{n}, p)=1$ and $\operatorname{gcd}(\hat{n}, q)=1$; in particular, $\hat{n}, u, v$ are pairwise coprime so that $\hat{n} \leq n / u v$. We set $\hat{Y}=\mathbb{S}^{3} / H$, which is either $\mathbb{S}^{3}$ or a lens space. With this understood, let $f: \hat{Y} \rightarrow \hat{Y}$ be a periodic diffeomorphism such that $Y=\hat{Y} /\langle f\rangle$.

Lemma 6.1 For any singular circle $\gamma$ of $Y$, say the one defined by $z_{2}=0$ which has multiplicity $u$, we let $\hat{\gamma}$ be the preimage of $\gamma$ in $\hat{Y}$. Then there exist a periodic diffeomorphism $f^{\prime}: \hat{Y} \rightarrow \widehat{Y}$ and an isotopy $f_{t}: \hat{Y} \rightarrow \widehat{Y}$ between $f$ and $f^{\prime}$, such that:

- The restriction of $f_{t}$ on $\hat{\gamma}$ is independent of $t$ (in particular, $f=f^{\prime}$ on $\hat{\gamma}$ ).

- $f^{\prime}$ is free on $\hat{\gamma}$ so that the image of $\hat{\gamma}$ in $Y^{\prime}=\hat{Y} /\left\langle f^{\prime}\right\rangle$ is not a singular circle.

- When $\hat{Y}=\mathbb{S}^{3}$, one can arrange $f^{\prime}$ such that $Y^{\prime}$ is the lens space $L(n / u, 1)$.

Proof We first consider the case where $\hat{n}>1$. Set $p^{\prime}=p / u$, let $u^{\prime}$ be the unique integer satisfying $u u^{\prime} \equiv 1(\bmod \hat{n})$ and $0<u^{\prime}<\hat{n}$, and consider the following action of a cyclic subgroup $G^{\prime} \subset \mathrm{SO}(4)$ of order $n^{\prime}=n / u$, given by

$$
\delta \cdot\left(z_{1}, z_{2}\right)=\left(\delta^{p^{\prime}} z_{1}, \delta^{q u^{\prime}} z_{2}\right),
$$

where $\delta=\exp \left(2 \pi i / n^{\prime}\right)$ is an $n^{\prime \text { th }}$ root of unity. Note that since $\lambda^{n / \hat{n}} \cdot\left(z_{1}, z_{2}\right)=$ $\delta^{n^{\prime} u / \hat{n}} \cdot\left(z_{1}, z_{2}\right), H=\left\langle\lambda^{n / \hat{n}}\right\rangle=\left\langle\delta^{n^{\prime} / \hat{n}}\right\rangle$ is also a subgroup of $G^{\prime}$.

There is a $k$ with $\operatorname{gcd}(n, k)=1$ such that $f: \hat{Y} \rightarrow \hat{Y}$ is represented by the $H-$ equivariant map $F:\left(z_{1}, z_{2}\right) \mapsto \lambda^{k} \cdot\left(z_{1}, z_{2}\right)$. We shall consider the $H$-equivariant map $F^{\prime}:\left(z_{1}, z_{2}\right) \mapsto \delta^{k} \cdot\left(z_{1}, z_{2}\right)$, which has the following properties: (i) $F=F^{\prime}$ on $\left\{\left(z_{1}, 0\right)|| z_{1} \mid=1\right\}$, (ii) there is an $H$-equivariant isotopy $F_{t}$ between $F$ and $F^{\prime}$ which is constant in $t$ on $\left\{\left(z_{1}, 0\right)|| z_{1} \mid=1\right\}$. For instance, $F_{t}:\left(z_{1}, z_{2}\right) \mapsto\left(\delta^{k p^{\prime}} z_{1}, \theta_{t} z_{2}\right)$, 
where $\theta_{t}=\exp \left(2 t k q u^{\prime} \pi i / n^{\prime}+2(1-t) k q \pi i / n\right), 0 \leq t \leq 1$. Let $f^{\prime}, f_{t}$ be the descendant of $F^{\prime}, F_{t}$ to $\hat{Y}$ respectively. Then clearly $f_{t}$ is an isotopy between $f$ and $f^{\prime}$ that is constant on $\hat{\gamma}=\left\{\left(z_{1}, 0\right)|| z_{1} \mid=1\right\} / H$, and $f^{\prime}$ is free on $\hat{\gamma}$ so that the image of $\hat{\gamma}$ in $Y^{\prime}=\hat{Y} /\left\langle f^{\prime}\right\rangle$ is not a singular circle. This finishes the proof for the case where $\hat{n}>1$.

Now suppose $\hat{n}=1$, which means that $H$ is trivial. Then instead, we consider the following action of a cyclic subgroup $G^{\prime} \subset \mathrm{SO}(4)$ of order $n^{\prime}=n / u$, given by

$$
\delta \cdot\left(z_{1}, z_{2}\right)=\left(\delta^{p^{\prime}} z_{1}, \delta^{p^{\prime}} z_{2}\right) .
$$

The rest of the argument is the same, with $H \subset G^{\prime}$ trivially true. Note that in this case, $Y^{\prime}=\mathbb{S}^{3} /\left\langle f^{\prime}\right\rangle=\mathbb{S}^{3} / G^{\prime}=L(n / u, 1)$. This finishes the proof of Lemma 6.1.

As an immediate corollary of Lemma 6.1, we obtain the following classification of fixed-point free smooth $\mathbb{S}^{1}$-four-manifolds whose fundamental group is isomorphic to the fundamental group of a Klein bottle.

Theorem 6.2 Let $X$ be a fixed-point free smooth $\mathbb{S}^{1}-$ four-manifold such that $\pi_{1}(X)$ is isomorphic to the fundamental group of a Klein bottle. Then $X$ is diffeomorphic to the quotient of $T^{2} \times \mathbb{S}^{2}$ by the involution $\tau$, where

$$
\tau:(x, y, z) \mapsto(-x, \bar{y},-z) \quad \text { for } x, y \in \mathbb{S}^{1} \subset \mathbb{C} \text { and } z \in \mathbb{S}^{2} \subset \mathbb{R}^{3} .
$$

Proof As $\pi_{1}(X)$ is isomorphic to the fundamental group of a Klein bottle, it has the following presentation: $\pi_{1}(X)=\left\{c, t \mid t c t^{-1}=c^{-1}\right\}$. Clearly the center $z\left(\pi_{1}(X)\right)$ is the infinite cyclic subgroup generated by $t^{2}$. By Theorem 1.4, the $\mathbb{S}^{1}$-action is injective. We let $\pi: X \rightarrow Y$ be the corresponding orbit map. Let $m>0$ be the multiplicity of the homotopy class of a regular fiber of $\pi$ in $z\left(\pi_{1}(X)\right)$. Then

$$
\pi_{1}^{\text {orb }}(Y)=\left\{c, t \mid t c t^{-1}=c^{-1}, t^{2 m}=1\right\} .
$$

Let $\hat{Y}$ be the regular covering of $Y$ corresponding to the infinite normal cyclic subgroup generated by $c$. Since $\hat{Y}$ is good and its fundamental group is torsion-free, $\widehat{Y}$ must be a 3-manifold, and clearly, $\hat{Y}=\mathbb{S}^{1} \times \mathbb{S}^{2}$. The corresponding group of deck transformations on $\hat{Y}$ is cyclic of order $2 m$ and is generated by $t$, which sends $c \in \pi_{1}(\hat{Y})$ to $-c$. By Lemma 2.5, $Y$ is diffeomorphic to either $\mathbb{R P}_{m}^{3} \#_{m} \mathbb{R P}_{m}^{3}, \mathbb{R P}_{m}^{3} \#_{m} \widetilde{R P}^{3}{ }_{m}$, or $\widetilde{\mathbb{R P}^{3}}{ }_{m} \#_{m} \widetilde{\mathbb{R P}^{3}}{ }_{m}$. Consequently, $X$ is fiber-sum-decomposed into $X_{1}$ and $X_{2}$ along $N$, with $\pi_{i}: X_{i} \rightarrow Y_{i}, i=1,2$, where each of $Y_{1}$ and $Y_{2}$ is either $\mathbb{R P}_{m}^{3}$ or $\mathbb{R P}^{3}{ }_{m}$, and $\pi: N \rightarrow \Sigma$ where $\Sigma$ intersects the singular circle of multiplicity $m$ in $Y$.

There are $\hat{Y}_{i}$ and periodic diffeomorphisms $f_{i}$ such that $Y_{i}=\hat{Y}_{i} /\left\langle f_{i}\right\rangle$ and $X_{i}$ is the mapping torus of $f_{i}$, where $i=1,2$. We apply Lemma 6.1 to $Y_{i}, \hat{Y}_{i}$, and $f_{i}$, with $\gamma$ 
being the singular circle of multiplicity $m$. We claim that in either case, ie $Y_{i}=\mathbb{R P}_{m}^{3}$ or $\widetilde{\mathbb{R P}^{3}}{ }_{m}, \hat{Y}_{i}$ must be $\mathbb{S}^{3}$, ie $\hat{n}=1$. For the case where $Y_{i}=\widetilde{\mathbb{R P}^{3}}{ }_{m}$, it follows from the fact that $\widetilde{\mathbb{R P}^{3}} m$ has two singular circles with multiplicities 2 and $m$, respectively, so that $\hat{n} \leq n / u v=2 m / 2 m=1$. For the case where $Y_{i}=\mathbb{R P}_{m}^{3}$, a similar argument shows that $\hat{n} \leq 2$. Continuing using the notations in Lemma 6.1, we have, in this case, $p=m, q=1, n^{\prime}=2$, and $f_{i}^{\prime}$ is given by multiplication by $\delta$. If $\hat{n}=2$ and, therefore, $\hat{Y}_{i}=\mathbb{R P}^{3}, f_{i}^{\prime}$ is the identity map on $\hat{Y}_{i}$. Consequently, as the mapping torus of $f_{i}^{\prime}$, $X_{i}$ is diffeomorphic to $\mathbb{S}^{1} \times \widehat{Y}_{i}$, and $\pi_{1}(X)$ contains a torsion subgroup of $\mathbb{Z}_{2}$ coming from $\pi_{1}\left(\hat{Y}_{i}\right)$. But this contradicts the fact that $\pi_{1}(X)$ is isomorphic to the $\pi_{1}$ of a Klein bottle. Hence $\hat{Y}_{i}=\mathbb{S}^{3}$ in both cases. We conclude by observing that each $X_{i}$ is the mapping torus of the antipodal map on $\mathbb{S}^{3}$. We denote by $\pi_{i}^{\prime}: X_{i} \rightarrow \mathbb{R P}^{3}$ the corresponding Seifert-type $\mathbb{S}^{1}$-fibration.

Finally, by the property in Lemma 6.1 that the restriction of $f_{t}$ on $\hat{\gamma}$ is independent of $t$, it is easily seen that the Seifert-type $\mathbb{S}^{1}$-fibrations $\pi_{i}: X_{i} \rightarrow Y_{i}$ and $\pi_{i}^{\prime}: X_{i} \rightarrow \mathbb{R P}^{3}$ are identical on the mapping torus of $f=f^{\prime}: \hat{\gamma} \rightarrow \hat{\gamma}$. It follows easily that $X$ is also fiber-sum-decomposed into $X_{1}$ and $X_{2}$ along $N$, with $\pi_{i}^{\prime}: X_{i} \rightarrow \mathbb{R P}^{3}$ on each factor $X_{i}$. Theorem 6.2 follows easily.

Theorem 1.1 follows immediately from the following theorem.

Theorem 6.3 Let $G$ be a finitely presented group such that:

(i) $\operatorname{rank} z(G)=1$.

(ii) $G$ is single-ended and is not isomorphic to the $\pi_{1}$ of a Klein bottle.

(iii) Any canonical JSJ decomposition of $G$ contains a vertex subgroup which is not isomorphic to an HNN extension of a finite cyclic group.

Let $S_{G}$ be the set of equivariant diffeomorphism classes of orientable, fixed-point free, smooth $\mathbb{S}^{1}$-four-manifolds $X$ such that $\pi_{1}(X)=G$. Then there exists a constant $C>0$, depending only on $G$, such that $\# S_{G}<C$.

Proof Let $X$ be an orientable, fixed-point free, smooth $\mathbb{S}^{1}$-four-manifold such that $\pi_{1}(X)=G$. Since $G$ is single-ended, it follows easily from Theorem 1.4 that any fixed-point free $\mathbb{S}^{1}$-action on $X$ must be injective. Thus, any fixed-point free $\mathbb{S}^{1}$ action on $X$ is associated with a canonical fiber-sum decomposition. Suppose $X$ is decomposed into factors $X_{i}$ along $N_{j}$. For convenience we shall fix an orientation of $X$, which is the one induced from the fiber-sum decomposition. Then the following data completely determine the oriented equivariant diffeomorphism class of $X$ : 
(i) The isomorphism class of the underlying graph of $\Lambda$.

(ii) For each pair of $i, j$ such that $N_{j} \subset X_{i}$, the fiber-preserving isotopy class of embeddings of $N_{j}$ in $X_{i}$ for each fixed oriented, fiber-preserving diffeomorphism class of $X_{i}$.

(iii) For each $i$, the oriented, fiber-preserving diffeomorphism class of $X_{i}$.

These data are subject to the following constraints: the cardinalities of $\left\{X_{i}\right\}$ and $\left\{N_{j}\right\}$ and the conjugacy classes of subgroups $\pi_{1}\left(X_{i}\right)$ and $\pi_{1}\left(N_{j}\right)$ in $G$ are determined by $G$; see Proposition 3.5. With this understood, our aim is to show that the number of objects in each of (i), (ii), and (iii) is bounded by a constant depending only on $G$.

The number of objects in (i) is clearly bounded by a constant depending only on $G$, since the cardinalities of $\left\{X_{i}\right\}$ and $\left\{N_{j}\right\}$ are fixed by $G$. For the objects in (ii) and (iii), where an index $i$ is being fixed, we shall discuss separately according to the following three cases, (a) rank $z\left(\pi_{1}\left(X_{i}\right)\right)>1$, (b) $\pi_{1}\left(X_{i}\right)$ is single-ended with rank $z\left(\pi_{1}\left(X_{i}\right)\right)=1$, (c) $\pi_{1}\left(X_{i}\right)$ is double-ended.

Note that the number of objects in (ii) is bounded by the number of singular circles of $Y_{i}$ plus one, so we need to show that, for each $i$, the number of singular circles of $Y_{i}$ is bounded by a constant depending only on $G$. With this understood, consider case (a) where $X_{i}$ is a Seifert-type $T^{2}$-fibration over a 2 -orbifold $B_{i}$ with infinite $\pi_{1}^{\text {orb }}$. As shown in the proof of Theorem 1.6(1), $B_{i}$ is uniquely determined by $\pi_{1}\left(X_{i}\right)$, hence by $G$. On the other hand, $Y_{i}$ is Seifert fibered over $B_{i}$, so that the number of singular circles of $Y_{i}$ is bounded by the number of singular points of $B_{i}$, which depends only on $G$. In case (b), $Y_{i}$ is uniquely determined by $\pi_{1}\left(X_{i}\right)$ as shown in the proof of Theorem 1.6(1), hence the number of singular circles of $Y_{i}$ depends only on $G$. In case (c), $\pi_{1}^{\text {orb }}\left(Y_{i}\right)$ is finite. The Geometrization theorem implies that $Y_{i}$ is spherical. Since the singular set of $Y_{i}$ consists of a union of embedded circles, the work of Dunbar in [15] shows that $Y_{i}=\mathbb{S}^{3} / G_{i}$, where $G_{i}$ is a subgroup of $\mathrm{SO}(4)$ which preserves a Hopf fibration. It follows easily that the number of singular components of $Y_{i}$ is universally bounded (say by 4 ). This shows that the number of objects in (ii) is bounded by a constant depending only on $G$.

Finally, we examine the boundedness of the number of objects in (iii). In case (a), the diffeomorphism class of $X_{i}$ is uniquely determined by $\pi_{1}\left(X_{i}\right)$ (see Theorem 1.6(1)); however, the Seifert-type $\mathbb{S}^{1}$-fibration $\pi_{i}: X_{i} \rightarrow Y_{i}$ has infinitely many choices, one for each primitive element of $z\left(\pi_{1}\left(X_{i}\right)\right)$. With this understood, note that, by assumption, $z(G)$ has rank 1 , so there is only one possible choice for the regular fiber class of $\pi_{i}$ in $z\left(\pi_{1}\left(X_{i}\right)\right)$. This shows that $\pi_{i}: X_{i} \rightarrow Y_{i}$ is uniquely determined by $G$ in this case. In case (b), both $X_{i}$ and $\pi_{i}$ are uniquely determined by $\pi_{1}\left(X_{i}\right)$, as shown in the proof of Theorem 1.6(1), and hence are also determined by $G$. 
Lastly, we consider case (c). By Theorem 1.6(2), $X_{i}$ is the mapping torus of a periodic diffeomorphism $f_{i}: \hat{Y}_{i} \rightarrow \hat{Y}_{i}$ of an elliptic 3-manifold. It follows from the proof of Theorem 1.6(2) that the number of diffeomorphism classes of $X_{i}$ is bounded by a constant depending only on $\pi_{1}\left(X_{i}\right)$. In order to bound the number of fiber-preserving diffeomorphism classes, we shall employ the rigidity theorem of injective Seifert fibered space constructions as in the proof of Theorem 1.6(1), with $k=1$ and $W=\mathbb{S}^{3}$. With this understood, it is clear that it suffices to show that the number of possible short exact sequences $1 \rightarrow \Gamma \rightarrow \pi \rightarrow Q \rightarrow 1$ involved in the argument is bounded by a constant depending only on $G$. Equivalently, we will show that the multiplicity of the homotopy class of a regular fiber of $\pi_{i}$ in $z\left(\pi_{1}\left(X_{i}\right)\right)$ is bounded by a constant depending only on $G$.

Denote by $h$ the homotopy class of a regular fiber. Since the conjugacy classes of the subgroups $\pi_{1}\left(X_{i}\right)$ in $G$ depend only on $G$, it follows easily that it suffices to bound the multiplicity of $h$ in $z\left(\pi_{1}(X)\right)$. With this understood, we observe that since for each $j, z\left(\pi_{1}(X)\right) \subset \pi_{1}\left(N_{j}\right)$, the multiplicity of $h$ in $z\left(\pi_{1}(X)\right)$ is bounded by the multiplicity of $h$ in $\pi_{1}\left(N_{j}\right)$ for every $j$, which equals 1 if $\Sigma_{j}$ is an ordinary 2-sphere, and equals the multiplicity of the singular circle of $Y$ that $\Sigma_{j}$ intersects otherwise. In particular, if one of the $\Sigma_{j}$ is an ordinary 2-sphere, or one of the $Y_{i}$ has infinite fundamental group, we are done for (iii). (Note that, since $G$ is single-ended, there is at least one $N_{j}$ if case (c) is valid.)

Suppose $\pi_{1}^{\text {orb }}\left(Y_{i}\right)$ is finite for each $i$ and $\Sigma_{j}$ is a football for each $j$. Again, since the singular set of $Y_{i}$ consists of a union of embedded circles, the work of Dunbar in [15] shows that $Y_{i}=\mathbb{S}^{3} / G_{i}$ for a finite subgroup $G_{i}$ of $\mathrm{SO}(4)$ that preserves a Hopf fibration. It follows that $X_{i}$ is the mapping torus of a periodic diffeomorphism $f_{i}: \hat{Y}_{i} \rightarrow \hat{Y}_{i}$, where $\hat{Y}_{i}$ has a Seifert fibration induced from the Hopf fibration and $f_{i}$ preserves the Seifert fibration on $\hat{Y}_{i}$. By the assumption (iii), there is a $Y_{i}$ such that $\pi_{1}\left(\hat{Y}_{i}\right)$ is nonabelian. With the following lemma (Lemma 6.4), we finish the proof by observing that $\pi_{1}\left(\hat{Y}_{i}\right)$ is completely determined by $\pi_{1}\left(X_{i}\right)$, which depends only on $G$.

Lemma 6.4 Let $\hat{Y}$ be an elliptic 3-manifold with nonabelian fundamental group, and let $\pi: \hat{Y} \rightarrow B$ be the unique Seifert fibration on $\hat{Y}$. Suppose $f: \hat{Y} \rightarrow \hat{Y}$ is an orientation-preserving periodic diffeomorphism that preserves $\pi$. Then the multiplicity of any singular circle of the 3-orbifold $Y=\hat{Y} /\langle f\rangle$ is bounded by a constant depending only on the multiplicities of the singular points of $B$.

Proof For any singular circle $\gamma$ in $Y$, the multiplicity of $\gamma$ equals the order of its isotropy subgroup. Let $f_{\gamma}$ be a generator of the isotropy subgroup, which is given 
by $f^{k}$ for some $k$. Since $f: \hat{Y} \rightarrow \hat{Y}$ preserves $\pi: \hat{Y} \rightarrow B$, so does $f_{\gamma}$, and there is an induced periodic diffeomorphism $\bar{f}_{\gamma}: B \rightarrow B$ of the 2-orbifold $B$.

Since $\pi_{1}(\hat{Y})$ is nonabelian, $B$ is a turnover with multiplicities $(2,2, n),(2,3,3)$, $(2,3,4)$, or $(2,3,5)$. We shall discuss according to the following cases: (i) $\bar{f}_{\gamma}$ is trivial, (ii) $\bar{f}_{\gamma}$ is nontrivial.

Suppose $\bar{f}_{\gamma}$ is trivial. Then $f_{\gamma}$ acts as a rotation on each fiber of $\pi: \hat{Y} \rightarrow B$. It follows easily that $\gamma$ must be an exceptional fiber of $\pi$, and the order of $f_{\gamma}$ is a divisor of the multiplicity of the singular point $\pi(\gamma) \in B$.

Suppose $\bar{f}_{\gamma}$ is nontrivial. Then there are two possibilities: (a) $\bar{f}_{\gamma}$ is orientationpreserving, (b) $\bar{f}_{\gamma}$ is orientation-reversing. In case (a), the order of $\bar{f}_{\gamma}$ is either 2 or 3 , and $\bar{f}_{\gamma}$ has two isolated fixed-points. Moreover, $\gamma$ must be the fiber over one of the fixed-points of $\bar{f}_{\gamma}$. It follows easily that the multiplicity of $\gamma$ equals the order of $\overline{f_{\gamma}}$, which is at most 3 . In case (b), $\bar{f}_{\gamma}$ must be a reflection over a great circle in $B$ because $\bar{f}_{\gamma}$ has a nonempty fixed-point (which contains $\pi(\gamma)$, for instance). Since $f$ is orientation-preserving, $f_{\gamma}$ must be a reflection on the fibers over the great circle fixed under $\bar{f}_{\gamma}$. It follows that the multiplicity of $\gamma$ equals 2 in this case.

Proof of Theorem 1.2 This result follows from Theorem 6.3 except in the following cases:

(a) $\operatorname{rank} z(G)>1$.

(b) $G$ is double-ended.

(c) $G$ is isomorphic to the $\pi_{1}$ of a Klein bottle.

(d) None of the above is true, and moreover, every vertex subgroup of a canonical JSJ decomposition of $G$ is an HNN extension of a finite cyclic group.

Cases (a), (c) are settled with the help of Theorems 4.3 and 6.2. Case (b) is settled by Theorem 1.4, Lemma 4.2, and Theorem 1.6. (Note that in case (b) where $G$ is double-ended, we appeal to Theorem 1.6(2), where we observe that when $X$ is a mapping torus of a periodic diffeomorphism of a lens space, the number of possible lens spaces is bounded by a constant depending only on $\pi_{1}(X)$.)

For case (d), we shall continue with the proof of Theorem 6.3, where we are left with the situation that $\pi_{1}\left(\hat{Y}_{i}\right)$ is finite cyclic for each $i$ and $\Sigma_{j}$ is a football for each $j$. Recall that $Y_{i}=\hat{Y}_{i} /\left\langle f_{i}\right\rangle$ for some periodic diffeomorphism $f_{i}$. Moreover, there is a Seifert fibration $\operatorname{pr}_{i}: \hat{Y}_{i} \rightarrow B_{i}$ which is induced from a Hopf fibration and is preserved under $f_{i}$. 
We shall analyze the multiplicities of the singular circles in $Y_{i}$. To this end, let $\gamma$ be a singular circle and $f_{\gamma}$ be a generator of its isotropy subgroup. Denote by $\bar{f}_{\gamma}: B_{i} \rightarrow B_{i}$ the induced map. If $\bar{f}_{\gamma}$ is orientation-reversing, then as we showed in the proof of Lemma 6.4, the multiplicity of $\gamma$ is 2 . If $\bar{f}_{\gamma}$ is orientation-preserving and switches the two singular points of $B_{i}$, then the multiplicity of $\gamma$ is also 2 , as we argued in the proof of Lemma 6.4. In the remaining cases where $\bar{f}_{\gamma}$ is either trivial or fixes the two singular points of $B_{i}$, or $B_{i}$ has no singular points at all, the multiplicity of $\gamma$ may not be bounded by a constant depending only on $G$, and we need to deal with it differently.

Note that in either of the remaining cases, $Y_{i}=\mathbb{S}^{3} / G_{i}$ for a finite subgroup $G_{i}$ of $\mathrm{SO}(4)$, which is given by

$$
\lambda \cdot\left(z_{1}, z_{2}\right)=\left(\lambda^{p_{i}} z_{1}, \lambda^{q_{i}} z_{2}\right),
$$

where $\lambda=\exp \left(2 \pi i / n_{i}\right)$ is a $n_{i}^{\text {th }}$ root of unity and $\operatorname{gcd}\left(n_{i}, p_{i}, q_{i}\right)=1$. Set

$$
u_{i}=\operatorname{gcd}\left(n_{i}, p_{i}\right) \quad \text { and } \quad v_{i}=\operatorname{gcd}\left(n_{i}, q_{i}\right) .
$$

Then $Y_{i}$ has at most two singular circles of multiplicities $u_{i}$ and $v_{i}$, respectively. Furthermore, if $\Sigma_{j}$ intersects the singular circle of multiplicity $u_{i}$ (resp. $v_{i}$ ), the index of $\pi_{1}\left(N_{j}\right)$ in $\pi_{1}\left(X_{i}\right)$ is $n_{i} / u_{i}$ (resp. $\left.n_{i} / v_{i}\right)$. Consequently, if both singular circles of $Y_{i}$ are intersected by $\Sigma_{j}$ for some $j$, then $u_{i} \leq n_{i} / v_{i}$ and $v_{i} \leq n_{i} / u_{i}$ are both bounded by a constant depending only on $G$; see Proposition 3.5. Clearly, we are done for (iii) in the proof of Theorem 6.3 if there exists a $Y_{i}$ for which such a situation occurs.

We are left to examine the case where, for each $i$, there is exactly one singular circle of $Y_{i}$ which is intersected by $\Sigma_{j}$ for some $j$. In this case, we shall apply Lemma 6.1 and change the Seifert-type $\mathbb{S}^{1}$-fibrations $\pi_{i}: X_{i} \rightarrow Y_{i}$ in the fiber-sum decomposition of $X$ to $\pi_{i}^{\prime}: X_{i} \rightarrow Y_{i}^{\prime}$. Note that with the new fibrations $\pi_{i}^{\prime}$, each $N_{j}$ is fibered over an ordinary 2-sphere. Consequently, up to suitable modifications of the Seifert-type $\mathbb{S}^{1}$-fibrations, the number of objects in (iii) in the proof of Theorem 6.3 is bounded by a constant depending only on $G$, from which Theorem 1.2 follows.

Acknowledgments The author is in debt to Slawomir Kwasik for many helpful conversations throughout the course of the work. This work was partly done during the author's visit at the Institute for Advanced Study, and he wishes to thank the institute for its hospitality and financial support. Finally, the author thanks the referee for a very careful reading of the article and for the many suggestions which have improved considerably the exposition of the paper. The author is partially supported by NSF grant DMS-1065784. 


\section{References}

[1] K Asano, Homeomorphisms of prism manifolds, Yokohama Math. J. 26 (1978) 19-25 MR524878

[2] S J Baldridge, Seiberg-Witten invariants, orbifolds, and circle actions, Trans. Amer. Math. Soc. 355 (2003) 1669-1697 MR1946410

[3] S Baldridge, Seiberg-Witten vanishing theorem for $S^{1}$-manifolds with fixed points, Pacific J. Math. 217 (2004) 1-10 MR2105762

[4] M Boileau, B Leeb, J Porti, Geometrization of 3-dimensional orbifolds, Ann. of Math. 162 (2005) 195-290 MR2178962

[5] M Boileau, S Maillot, J Porti, Three-dimensional orbifolds and their geometric structures, Panoramas et Synthèses 15, Soc. Math. France, Paris (2003) MR2060653

[6] M Boileau, J-P Otal, Scindements de Heegaard et groupe des homéotopies des petites variétés de Seifert, Invent. Math. 106 (1991) 85-107 MR1123375

[7] F Bonahon, Difféotopies des espaces lenticulaires, Topology 22 (1983) 305-314 MR710104

[8] S Cappell, S Weinberger, M Yan, Closed aspherical manifolds with center, J. Topol. 6 (2013) 1009-1018 MR3145148

[9] A Casson, D Jungreis, Convergence groups and Seifert fibered 3-manifolds, Invent. Math. 118 (1994) 441-456 MR1296353

[10] W Chen, On a notion of maps between orbifolds, II: Homotopy and CW-complex, Commun. Contemp. Math. 8 (2006) 763-821 MR2274941

[11] W Chen, Seiberg-Witten invariants of 3-orbifolds and non-Kähler surfaces, J. Gökova Geom. Topol. GGT 6 (2012) 1-27 MR3011643

[12] W Chen, Hurwitz-type bound, knot surgery, and smooth $\mathbb{S}^{1}$-four-manifolds, Math. Z. 276 (2014) 267-279 MR3150204

[13] W Dicks, M J Dunwoody, Groups acting on graphs, Cambridge Studies in Advanced Mathematics 17, Cambridge Univ. Press (1989) MR1001965

[14] J Dinkelbach, B Leeb, Equivariant Ricci flow with surgery and applications to finite group actions on geometric 3-manifolds, Geom. Topol. 13 (2009) 1129-1173 MR2491658

[15] W D Dunbar, Nonfibering spherical 3-orbifolds, Trans. Amer. Math. Soc. 341 (1994) 121-142 MR1118824

[16] R Fintushel, Locally smooth circle actions on homotopy 4-spheres, Duke Math. J. 43 (1976) 63-70 MR0394716

[17] R Fintushel, Circle actions on simply connected 4-manifolds, Trans. Amer. Math. Soc. 230 (1977) 147-171 MR0458456 
[18] R Fintushel, Classification of circle actions on 4-manifolds, Trans. Amer. Math. Soc. 242 (1978) 377-390 MR496815

[19] R Fintushel, R J Stern, Immersed spheres in 4-manifolds and the immersed Thom conjecture, Turkish J. Math. 19 (1995) 145-157 MR1349567

[20] D Gabai, Convergence groups are Fuchsian groups, Ann. of Math. 136 (1992) 447-510 MR1189862

[21] A Haefliger, Homotopy and integrability, from: "Manifolds", (N H Kuiper, editor), Lecture Notes in Mathematics 197, Springer, Berlin (1971) 133-163 MR0285027

[22] A Haefliger, Groupoïdes d'holonomie et classifiants, from: "Transversal structure of foliations", 116 (1984) 70-97 MR755163

[23] A Hatcher, On the diffeomorphism group of $S^{1} \times S^{2}$, Proc. Amer. Math. Soc. 83 (1981) 427-430 MR624946

[24] J Hempel, 3-manifolds, Ann. of Math. Studies 86, Princeton Univ. Press (1976) MR0415619

[25] J A Hillman, Four-manifolds, geometries and knots, Geometry \& Topology Monographs 5 (2002) MR1943724

[26] C Hodgson, J H Rubinstein, Involutions and isotopies of lens spaces, from: “Knot theory and manifolds", (D Rolfsen, editor), Lecture Notes in Math. 1144, Springer, Berlin (1985) 60-96 MR823282

[27] B Kleiner, J Lott, Notes on Perelman's papers arXiv:math.DG/0605667

[28] K B Lee, F Raymond, The role of Seifert fiber spaces in transformation groups, from: "Group actions on manifolds", (R Schultz, editor), Contemp. Math. 36, Amer. Math. Soc. (1985) 367-425 MR780974

[29] A M Macbeath, Geometrical realization of isomorphisms between plane groups, Bull. Amer. Math. Soc. 71 (1965) 629-630 MR0177110

[30] W Magnus, A Karrass, D Solitar, Combinatorial group theory: presentations of groups in terms of generators and relations, Interscience, New York (1966) MR0207802

[31] D McCullough, A Miller, Manifold covers of 3-orbifolds with geometric pieces, Topology Appl. 31 (1989) 169-185 MR994409

[32] W H Meeks, III, P Scott, Finite group actions on 3-manifolds, Invent. Math. 86 (1986) 287-346 MR856847

[33] W H Meeks, III, S T Yau, Topology of three-dimensional manifolds and the embedding problems in minimal surface theory, Ann. of Math. 112 (1980) 441-484 MR595203

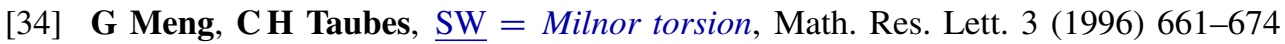
MR1418579 
[35] PS Pao, Nonlinear circle actions on the 4-sphere and twisting spun knots, Topology 17 (1978) 291-296 MR508892

[36] G Perelman, The entropy formula for the Ricci flow and its geometric applications arXiv:math/0211159

[37] C Petronio, Spherical splitting of 3-orbifolds, Math. Proc. Cambridge Philos. Soc. 142 (2007) 269-287 MR2314601

[38] E Rips, Z Sela, Cyclic splittings of finitely presented groups and the canonical JSJ decomposition, Ann. of Math. 146 (1997) 53-109 MR1469317

[39] J H Rubinstein, On 3-manifolds that have finite fundamental group and contain Klein bottles, Trans. Amer. Math. Soc. 251 (1979) 129-137 MR531972

[40] J H Rubinstein, J S Birman, One-sided Heegaard splittings and homeotopy groups of some 3-manifolds, Proc. London Math. Soc. 49 (1984) 517-536 MR759302

[41] P Scott, The geometries of 3-manifolds, Bull. London Math. Soc. 15 (1983) 401-487 MR705527

[42] P Scott, T Wall, Topological methods in group theory, from: "Homological group theory", (C T C Wall, editor), London Math. Soc. Lecture Note Ser. 36, Cambridge Univ. Press (1979) 137-203 MR564422

[43] Z Sela, Structure and rigidity in (Gromov) hyperbolic groups and discrete groups in rank 1 Lie groups, II, Geom. Funct. Anal. 7 (1997) 561-593 MR1466338

[44] G A Swarup, On a theorem of C B Thomas, J. London Math. Soc. 8 (1974) 13-21 MR0341474

[45] C B Thomas, The oriented homotopy type of compact 3-manifolds, Proc. London Math. Soc. 19 (1969) 31-44 MR0248838

[46] V G Turaev, Towards the topological classification of geometric 3-manifolds, from: "Topology and geometry - Rohlin Seminar", (O Y Viro, editor), Lecture Notes in Math. 1346, Springer, Berlin (1988) 291-323 MR970081

[47] V Turaev, A combinatorial formulation for the Seiberg-Witten invariants of 3manifolds, Math. Res. Lett. 5 (1998) 583-598 MR1666856

[48] T Yoshida, Simply connected smooth 4-manifolds which admit nontrivial smooth $S^{1}$ actions, Math. J. Okayama Univ. 20 (1978) 25-40 MR0494173

Department of Mathematics and Statistics, University of Massachusetts Amherst, MA 01003, USA

wchen@math . umass . edu

Received: 26 March 2014 Revised: 10 January 2015 
